\title{
Preliminary Test Estimators of the Reliability Characteristics for the Three Parameters Burr XII Distribution \\ Based on Records
}

\author{
Ajit Chaturvedi $^{\mathrm{a}}$, Reza Arabi Belaghi ${ }^{\mathrm{b}}$ and Ananya Malhotra ${ }^{\mathrm{a},{ }^{*}}$ \\ ${ }^{\text {a }}$ Department of Statistics, Faculty of Mathematical Sciences, University of Delhi, Delhi - \\ 110007, INDIA \\ ${ }^{b}$ Department of Statistics, Faculty of Mathematical Sciences, University of Tabriz, Tabriz, \\ IRAN \\ *Corresponding Author Email: malhotra.ananya3@gmail.com
}

\begin{abstract}
Some improved estimators and confidence interval of the parametric functions are proposed based on records from three parameters Burr XII distribution. We propose preliminary test estimators (PTES) of the powers of the parameter and reliability functions based on uniformly minimum variance unbiased estimator (UMVUE), maximum likelihood estimator (MLE), best invariant estimator (MRE) and empirical Bayes estimator (EBE). We compare the performance of the proposed PTES with the usual estimators by studying their relative efficiencies based on Monte Carlo simulations. We also construct preliminary test confidence interval (PTCI) for the parameter and study its coverage probability and expected length. The results show that the proposed PTES dominate the usual estimators in a wide range of the parametric space. Also it is seen that the proposed PTCI have higher coverage probability while keeping the shorter width in some domain of parametric space. The paper ends up by analysing a real data set.
\end{abstract}

Keywords and Phrases: Three parameters Burr XII distribution; preliminary test estimator; preliminary test confidence interval; record values; Monte Carlo simulation

\section{Introduction}

In statistical inference, we often come across problems where some prior information on the parameters is available. Naturally, the validity of this information is questionable and hence it is required to perform a preliminary test on this constraint which gives rise to restricted models. Due to past knowledge or experience, the experimenter may be in a position to make an initial guess on some of the parameters of interest. In such cases, we can provide an improved estimator by incorporating this prior information on the parameters. The 
usage of this prior information on some or all of the parameters of a statistical distribution usually leads to an improved inferential study. The efficiency and validity of restricted models analysis is higher over a restricted parametric space induced by the constraints, while the same holds for unrestricted model analysis over the entire parametric space. Thus, an analysis resulting from restricted or unrestricted models may be subject to a loss in efficiency against the validity of constraints while choosing between two inferential techniques. Hence it is wise to adopt restricted estimation procedures when we have sufficient confidence in the prior information. Bancroft (1944) introduced the use of PTES and eventually further advancements were proposed by Saleh and Sen (1978), Saleh and Kibria (1993) and Belaghi et al. $(2014,2015)$.

The reliability function $R(t)$ is defined as the probability of failure-free operation until time $t$. Thus, if the random variable $(r v) X$ denotes the lifetime of an item or a system, then $R(t)=P(X>t)$. Another measure of reliability under stress-strength setup is the probability $P=P(X>Y)$, which represents the reliability of an item or a system of random strength $X$ subject to random stress $Y$. A lot of work has been done in the literature for the point estimation and testing of $R(t)$ and $P$ based on record values. For a brief review, one may refer to Chaturvedi and Malhotra $(2016,2017)$. In the present paper, we develop PTES for the reliability functions based on MLE and UMVUE and prove them to be more efficient estimators than their usual MLE and UMVUE.

Chandler (1952) introduced the concept of record values. Based on records, inferential procedures for the parameters of different distributions have been developed by Glick (1978), Nagaraja (1988a,1988b), Balakrishan, Ahsanullah and Chan (1995), Arnold, Balakrishan and Nagaraja (1992), Habibi Rad, Arghami and Ahmadi (2006), Arashi and Emadi (2008), Razmkhah and Ahmadi (2011), Belaghi et al. (2015) and others.

Many authors have studied the estimation of Burr type XII parameters. The two parameters Burr type XII distribution was first introduced by Burr (1942). A random variable $X$ is said to have a Burr type XII distribution with the shape parameters $c$ and $k$, if its cumulative distribution function $(c d f)$ and probability density function $(p d f)$ are of the following forms respectively:

$$
F(x ; k, c)=1-\left(1+x^{c}\right)^{-k} ; x>0, c, k>0
$$

and

$$
f(x ; k, c)=k c x^{c-1}\left(1+x^{c}\right)^{-(k+1)} ; x>0, c, k>0
$$


Some recent work on Burr XII model can be found in Belaghi and Noori (2016) and Belaghi et al. (2017). Belaghi et al. $(2014,2015)$ constructed PTES and PTCI based on record values for the Burr XII Model.

Shao (2004) expanded an extended the Burr type XII distribution to three parameters and used it for flood frequency analysis. Hogg and Klugman (1984) discussed a three parameters Burr XII distribution by introducing a scale parameter $\alpha$ to the Burr type XII distribution. This distribution has $p d f$ and $c d f$ of the following form respectively:

$f(x ; k, c, \alpha)=\frac{k c x^{c-1}}{\alpha}\left(1+\frac{x^{c}}{\alpha}\right)^{-(k+1)} ; x>0, k, c, \alpha>0$

and

$$
F(x ; k, c, \alpha)=1-\left(1+\frac{x^{c}}{\alpha}\right)^{-k} ; x>0, k, c, \alpha>0
$$

Let $X_{1}, X_{2}, \ldots$ be an infinite sequence of independent and identically distributed (iid) rvs from (1.1). An observation $X_{j}$ will be called an upper record value (or simply a record) if its value exceeds all the previous observations. Thus $X_{j}$ is a record if $X_{j}>X_{i}$ for every $i<j$. The record time sequence $\left\{T_{n}, n \geq 0\right\}$ is defined as

$$
\left\{\begin{array}{l}
T_{0}=1 \quad ; \text { with probability } 1 \\
T_{n}=\min \left\{j: X_{j}>X_{T_{n-1}}\right\} ; n \geq 1
\end{array}\right.
$$

and the record value sequence $\left\{R_{n}\right\}$ is then defined as

$$
R_{n}=X_{T_{n}} ; n=0,1,2, \ldots
$$

Following Chaturvedi and Malhotra (2017), the likelihood function of the first $n+1$ upper record values $R_{0}, R_{1}, R_{2}, \ldots, R_{n}$ is

$$
L\left(k \mid R_{0}, R_{1}, R_{2}, \ldots, R_{n}\right)=\left(\frac{k c}{\alpha}\right)^{n+1} \exp \left(-k \log \left(1+\frac{R_{n}^{c}}{\alpha}\right)\right) \prod_{i=0}^{n} \frac{R_{i}^{c-1}}{\left(1+\frac{R_{i}^{c}}{\alpha}\right)}
$$

For simplicity, we define

$$
U(x)=\log \left(1+\frac{x^{c}}{\alpha}\right)
$$

The rest of the paper is as follows. In Section 2, on the basis of records, we construct PTES based on MLE, UMVUE, MRE and EBE for the powers of the unknown parameter $k$ assuming the remaining parameters $c$ and $\alpha$ are known. We propose PTES based on MLE and UMVUE of the reliability functions $R(t)$ and $P$ for record values from (1.1). Then, bias and mean square error (MSE) of the proposed estimators are obtained. Relative efficiencies of the PTES are studied based on simulated data. PTCI of the unknown parameter $k$ are 
constructed based on UMVUE, MLE and MRE. Their coverage probability and expected length are derived for comparison with the equal tail confidence intervals (ETCI). In Section 3, we consider the case when all the parameters $k, c$ and $\alpha$ of the distribution in (1.1) are unknown and obtain the MLES of parameters and reliability functions. Following which we obtain asymptotic distributional bias (ADB) and asymptotic distributional mean square error (ADMSE) of the proposed estimators. Section 4 contains an illustrative example and finally in Section 5 we conclude our study.

\section{Estimation of Parametric Functions when $c$ and $\alpha$ are known}

For $p \in(-\infty, \infty), p \neq 0$ the MLE of $k^{p}$ is

$\hat{k}_{M L}^{p}=\left(\frac{n+1}{U\left(R_{n}\right)}\right)^{p}$

where $U\left(R_{n}\right)$ has gamma distribution with parameters $(n+1, k)$ [see Chaturvedi and Malhotra (2017)]. Further, the UMVUE of $k^{p}$ is

$\hat{k}_{U}^{p}=\left\{\begin{array}{r}\frac{\Gamma(n+1)}{\Gamma(n-p+1)}\left(U\left(R_{n}\right)\right)^{-p} ; p<n+1 \\ 0 ; \text { otherwise }\end{array}\right.$

For some constant $\omega$, minimising $\left(\omega \hat{k}_{U}-k\right)^{2}$ with respect to $\omega$ helps us to determine the MRE of $k$ denoted by $\hat{k}_{M R}$ and is obtained as follows:

$\hat{k}_{M R}=\frac{n-1}{n} \hat{k}_{U}$

Now, if we consider conjugate prior distribution of $k$ to be Gamma distribution with parameters $(\mu, v)$ and $p d f$

$$
\pi(k)=\frac{\mu^{v} k^{\nu-1}}{\Gamma(v)} e^{-\mu k} ; \mu, k>0 \text { and } v \text { is a positive integer }
$$

Then the posterior distribution of $k$ given $R_{0}, R_{1}, R_{2}, \ldots, R_{n}$ is

$\pi\left(k \mid R_{0}, R_{1}, R_{2}, \ldots, R_{n}\right)=\frac{\left(U\left(R_{n}\right)+\mu\right)^{n+v+1} k^{n+v}}{\Gamma(n+v+1)} e^{-k\left(U\left(R_{n}\right)+\mu\right)}$

Under squared error loss function, the Bayes estimator of $k^{p}$ is

$\hat{k}_{B}^{p}=\frac{\Gamma(n+v+p+1)}{\Gamma(n+v+1)}\left(U\left(R_{n}\right)+\mu\right)^{-p}$

Also, the marginal distribution of $R_{0}, R_{1}, R_{2}, \ldots, R_{n}$ given $\mu$ and $v$ is

$$
m\left(R_{0}, R_{1}, R_{2}, \ldots, R_{n} \mid \mu, v\right)=\int_{0}^{\infty} \pi(k) L\left(k \mid R_{0}, R_{1}, R_{2}, \ldots, R_{n}\right) d k
$$




$$
=\frac{\mu^{\nu} \Gamma(n+v+1)}{\left(U\left(R_{n}\right)+\mu\right)^{n+v+1} \Gamma(v)}\left(\frac{c}{\alpha}\right)^{n+1} \prod_{i=0}^{n} \frac{R_{i}^{c-1}}{\left(1+\frac{R_{i}^{c}}{\alpha}\right)}
$$

Taking the natural logarithm $l$ of the above marginal distribution, the MLE of $\mu$ and $v$ can be obtained from the solution of the following system of equations:

$$
\frac{\partial l}{\partial \mu}=\frac{v}{\mu}-\frac{n+v+1}{U\left(R_{n}\right)+\mu}=0
$$

and

$$
\frac{\partial l}{\partial v}=\log (\mu)+\frac{\partial}{\partial v}\left[\log \left(\frac{\Gamma(n+v+1)}{\Gamma(v)}\right)\right]-\log \left(U\left(R_{n}\right)+\mu\right)=0
$$

Denoting the MLE of $\mu$ and $v$ by $\hat{\mu}_{M L}$ and $\hat{v}_{M L}$ respectively, then there exists a relation between them given by

$$
\hat{\mu}_{M L}=\frac{U\left(R_{n}\right) \hat{v}_{M L}}{n+1}
$$

Therefore, from (2.5), the EBE of $k^{p}$ is

$\hat{k}_{E B}^{p}=\frac{\Gamma\left(n+\hat{v}_{M L}+p+1\right)}{\Gamma\left(n+\hat{v}_{M L}+1\right)}\left(U\left(R_{n}\right)+\hat{\mu}_{M L}\right)^{p}$

In the sequel we define four different PTES based on MLE, UMVUE, MRE and EBE of $k^{p}$ when it is suspected that $k$ may be equal to $k_{o}$. Often the information on the value of $k$ is available from the past knowledge or experiments. This non-sample prior information can be expressed in the form of the following group of hypotheses:

$$
\begin{aligned}
& H_{o}: k=k_{o} \\
& H_{1}: k \neq k_{o}
\end{aligned}
$$

Then based on classical hypothesis testing, the critical region is given by

$$
\left\{0<U\left(R_{n}\right)<l_{\mathrm{o}}\right\} \cup\left\{l_{\mathrm{o}}{ }^{\prime}<U\left(R_{n}\right)<\infty\right\}
$$

where $l_{o}$ and $l_{o}^{\prime}$ are obtained such that $l_{o}=\frac{\chi_{2(n+1)}^{2}\left(\frac{\varepsilon}{2}\right)}{2 k_{o}}, l_{\mathrm{o}}{ }^{\prime}=\frac{\chi_{2(n+1)}^{2}\left(1-\frac{\varepsilon}{2}\right)}{2 k_{o}}$ and $\varepsilon$ is the level of significance. Or, equivalently we reject $H_{O}$ if

$$
2 k_{o} U\left(R_{n}\right)<C_{2} \text { or } 2 k_{o} U\left(R_{n}\right)>C_{1}
$$

where $C_{1}=\chi_{2(n+1)}^{2}\left(1-\frac{\varepsilon}{2}\right)$ and $C_{2}=\chi_{2(n+1)}^{2}\left(\frac{\varepsilon}{2}\right)$.

Thus we define four different PTES for $k^{p}$ based on MLE, UMVUE, MRE and EBE respectively as

$$
\begin{aligned}
& \hat{k}_{M L}^{p P T}=\hat{k}_{M L}^{p}-\left(\hat{k}_{M L}^{p}-k_{o}^{p}\right) I(A) \\
& \hat{k}_{U}^{p^{P T}}=\hat{k}_{U}^{p}-\left(\hat{k}_{U}^{p}-k_{o}^{p}\right) I(A)
\end{aligned}
$$




$$
\begin{aligned}
& \hat{k}_{M R}^{P T}=\hat{k}_{M R}-\left(\hat{k}_{M R}-k_{o}\right) I(A) \\
& \hat{k}_{E B}^{p}{ }^{P T}=\hat{k}_{E B}^{p}-\left(\hat{k}_{E B}^{p}-k_{o}^{p}\right) I(A)
\end{aligned}
$$

where $I(A)$ is the indicator function of the set

$$
A=\left\{\chi_{2(n+1)}^{2}: C_{2} \leq \chi_{2(n+1)}^{2} \leq C_{1}\right\}
$$

From Chaturvedi and Malhotra (2017), the MLE and UMVUE of $R(t)$ are respectively given by

$$
\hat{R}(t)=\exp \left\{\frac{-(n+1) U(t)}{U\left(R_{n}\right)}\right\}
$$

and

$$
\tilde{R}(t)=\left\{\begin{array}{c}
{\left[1-\frac{U(t)}{U\left(R_{n}\right)}\right]^{n} ; U(t)<U\left(R_{n}\right)} \\
0 ; \text { otherwise }
\end{array}\right.
$$

Thus, we define two different PTES of $R(t)$ based on MLE and UMVUE as follows:

$$
\begin{aligned}
& \hat{R}(t)^{P T}=\hat{R}(t)-\left(\hat{R}(t)-R_{o}(t)\right) I(A) \\
& \tilde{R}(t)^{P T}=\tilde{R}(t)-\left(\tilde{R}(t)-R_{o}(t)\right) I(A)
\end{aligned}
$$

where $R_{o}(t)=e^{-k_{o} U(t)}$.

Let $X$ and $Y$ be two independent rvs from the three parameters Burr XII distribution with parameters $\left(k_{1}, c, \alpha\right)$ and $\left(k_{2}, c, \alpha\right)$ respectively. Let $R_{0}, R_{1}, \ldots, R_{n}$ be $n+1$ record values from distribution of $X$ and $R_{0}^{*}, R_{1}^{*}, \ldots, R_{m}^{*}$ be $m+1$ records from distribution of $Y$. Then, $P=\frac{k_{2}}{k_{1}+k_{2}}$. Suppose we want to test

$$
\begin{aligned}
& H_{\mathrm{o}}: P=P_{\mathrm{o}} \\
& H_{1}: P \neq P_{\mathrm{o}} .
\end{aligned}
$$

Note that $H_{\mathrm{o}}$ is equivalent to $k_{2}=\theta k_{1}$ where $\theta=\frac{P_{\mathrm{o}}}{1-P_{\mathrm{o}}}$. Thus, $H_{\mathrm{o}}: k_{2}=\theta k_{1}$ and $H_{1}: k_{2} \neq$ $\theta k_{1}$. It can be shown that, under $H_{\mathrm{o}}$,

$$
\hat{k}_{1}=\frac{n+m+2}{U\left(R_{n}\right)+\theta U\left(R_{m}^{*}\right)}
$$

and

$$
\hat{k}_{2}=\frac{\theta(n+m+2)}{U\left(R_{n}\right)+\theta U\left(R_{m}^{*}\right)}
$$

For a generic constant $C$, the likelihood function of $k_{1}$ and $k_{2}$ is

$$
L\left(k_{1}, k_{2} \mid R_{0}, R_{1}, \ldots, R_{n}, R_{0}^{*}, R_{1}^{*}, \ldots, R_{m}^{*}\right)=C k_{1}^{n+1} k_{2}^{m+1} \exp \left\{-\left(k_{1} U\left(R_{n}\right)+k_{2} U\left(R_{m}^{*}\right)\right)\right\}
$$

and thus, 


$$
\begin{aligned}
& \sup _{\Theta_{0}} L\left(k_{1}, k_{2} \mid R_{0}, R_{1}, \ldots, R_{n}, R_{0}^{*}, R_{1}^{*}, \ldots, R_{m}^{*}\right) \\
& =\frac{C}{\left\{U\left(R_{n}\right)+\theta U\left(R_{m}^{*}\right)\right\}^{n+m+2}} \exp \{-(n+m+2)\} ; \Theta_{0}=\left\{k_{1}, k_{2}: k_{2}=\theta k_{1}\right\}
\end{aligned}
$$

and

$$
\begin{aligned}
& \sup _{\Theta} L\left(k_{1}, k_{2} \mid R_{0}, R_{1}, \ldots, R_{n}, R_{0}^{*}, R_{1}^{*}, \ldots, R_{m}^{*}\right) \\
& =\frac{C}{\left\{U\left(R_{n}\right)\right\}^{n+1}\left\{U\left(R_{m}^{*}\right)\right\}^{m+1}} \exp \{-(n+m+2)\} ; \Theta=\left\{k_{1}, k_{2}: k_{1}>0, k_{2}>0\right\}
\end{aligned}
$$

Therefore, the Likelihood Ratio is

$$
\emptyset\left(R_{0}, R_{1}, \ldots, R_{n}, R_{0}^{*}, R_{1}^{*}, \ldots, R_{m}^{*}\right)=\frac{C\left\{\frac{U\left(R_{m}^{*}\right)}{U\left(R_{n}\right)}\right\}^{m+1}}{\left\{1+\frac{\theta U\left(R_{m}^{*}\right)}{U\left(R_{n}\right)}\right\}^{n+m+2}}
$$

Denoting by $F_{a, b}(\cdot)$, the $F$ - Statistic with $(a, b)$ degrees of freedom and using the fact that $\frac{U\left(R_{n}\right)}{U\left(R_{m}^{*}\right)} \sim \frac{(n+1) k_{2}}{(m+1) k_{1}} F_{2(n+1), 2(m+1)}$, the critical region is given by

$$
\left\{\frac{U\left(R_{n}\right)}{U\left(R_{m}^{*}\right)}<l_{1}\right\} \cup\left\{\frac{U\left(R_{n}\right)}{U\left(R_{m}^{*}\right)}>l_{1}^{\prime}\right\}
$$

where $l_{1}=\frac{\theta(n+1)}{(m+1)} F_{2(n+1), 2(m+1)}\left(\frac{\varepsilon}{2}\right)$ and $l_{1}^{\prime}=\frac{\theta(n+1)}{(m+1)} F_{2(n+1), 2(m+1)}\left(1-\frac{\varepsilon}{2}\right)$. Thus, we define two PTES of $P$ based on MLE and UMVUE of $P$ as follows:

$$
\begin{aligned}
& \hat{P}^{P T}=\hat{P}-\left(\hat{P}-P_{o}\right) I(B) \\
& \tilde{P}^{P T}=\tilde{P}-\left(\tilde{P}-P_{o}\right) I(B)
\end{aligned}
$$

where $I(B)$ is the indicator function of the set

$$
B=\left\{F_{2(n+1), 2(m+1)}: C_{4}<F_{2(n+1), 2(m+1)}<C_{3}\right\}
$$

Here, $C_{3}=F_{2(n+1), 2(m+1)}\left(1-\frac{\varepsilon}{2}\right), C_{4}=F_{2(n+1), 2(m+1)}\left(\frac{\varepsilon}{2}\right)$ and $\hat{P}$ and $\tilde{P}$ are the MLE and UMVUE of $P$ respectively as defined in Chaturvedi and Malhotra (2017) and are respectively given as

$$
\begin{aligned}
& \hat{P}=\frac{(m+1) U\left(R_{n}\right)}{(m+1) U\left(R_{n}\right)+(n+1) U\left(R_{m}^{*}\right)} \\
& \tilde{P}= \begin{cases}m \sum_{i=0}^{m-1}(-1)^{i} \frac{m ! n !}{(m-1-i) !(n+1+i) !}\left\{\frac{U\left(R_{n}\right)}{U\left(R_{m}^{*}\right)}\right\}^{i+1} & ; R_{n}<R_{m}^{*} \\
m \sum_{i=0}^{n}(-1)^{i} \frac{m ! n !}{(m+i) !(n-i) !}\left\{\frac{U\left(R_{m}^{*}\right)}{U\left(R_{n}\right)}\right\}^{i} & ; R_{n} \geq R_{m}^{*}\end{cases}
\end{aligned}
$$




\subsection{Bias and Mean Square Error}

In this sub-section, we derive the bias and mean square error (MSE) expressions for PTES based on UMVUE, MLE, MRE and EBE of $k^{p}$. For $=\frac{k}{k_{o}}$, we have

$$
\begin{aligned}
\operatorname{Bias}\left(\hat{k}_{U}^{p P T}\right) & =E\left[\hat{k}_{U}^{p}-\left(\hat{k}_{U}^{p}-k_{o}^{p}\right) I(A)-k^{p}\right] \\
& =k_{o}^{p}\left[\left\{H_{2 n+2}\left(\lambda C_{1}\right)-H_{2 n+2}\left(\lambda C_{2}\right)\right\}-\lambda^{p}\left\{H_{2(n-p+1)}\left(\lambda C_{1}\right)-H_{2(n-p+1)}\left(\lambda C_{2}\right)\right\}\right]
\end{aligned}
$$

where $H_{\gamma}($.$) stands for the c d f$ of $\chi^{2}$ distribution with $\gamma$ degrees of freedom.

Also, $\operatorname{MSE}\left(\hat{k}_{U}^{p^{P T}}\right)=\operatorname{Var}\left(k_{U}^{p^{P T}}\right)+\left[\operatorname{Bias}\left(\hat{k}_{U}^{p^{P T}}\right)\right]^{2}$

Since

$$
\begin{aligned}
\operatorname{Var}\left(k_{U}^{p P T}\right)=\operatorname{Var}\left(\hat{k}_{U}^{p}\right) & +\operatorname{Var}\left(\left(\hat{k}_{U}^{p}-k_{o}^{p}\right) I(A)\right)-2 \operatorname{Cov}\left(\hat{k}_{U}^{p},\left(\hat{k}_{U}^{p}-k_{o}^{p}\right) I(A)\right) \\
=\left(\lambda k_{o}\right)^{2 p} & {\left[\frac{\Gamma(n-2 p+1) \Gamma(n+1)}{\Gamma^{2}(n-p+1)}-1\right]+\left(\lambda k_{o}\right)^{2 p} \frac{\Gamma(n-2 p+1) \Gamma(n+1)}{\Gamma^{2}(n-p+1)}\left\{H_{2(n-2 p+1)}\left(\lambda C_{1}\right)-H_{2(n-2 p+1)}\left(\lambda C_{2}\right)\right\} } \\
& -\left(\lambda k_{o}\right)^{2 p}\left\{H_{2(n-p+1)}\left(\lambda C_{1}\right)-H_{2(n-p+1)}\left(\lambda C_{2}\right)\right\}^{2} \\
& +k_{o}^{2 p}\left\{H_{2 n+2}\left(\lambda C_{1}\right)-H_{2 n+2}\left(\lambda C_{2}\right)\right\}\left[1-\left\{H_{2 n+2}\left(\lambda C_{1}\right)-H_{2 n+2}\left(\lambda C_{2}\right)\right\}\right] \\
& -2 k_{o}^{2 p} \lambda^{p}\left\{H_{2(n-p+1)}\left(\lambda C_{1}\right)-H_{2(n-p+1)}\left(\lambda C_{2}\right)\right\}\left[1-\left\{H_{2 n+2}\left(\lambda C_{1}\right)-H_{2 n+2}\left(\lambda C_{2}\right)\right\}\right] \\
& -2\left(\lambda k_{o}\right)^{2 p} \frac{\Gamma(n-2 p+1) \Gamma(n+1)}{\Gamma^{2}(n-p+1)}\left\{H_{2(n-2 p+1)}\left(\lambda C_{1}\right)-H_{2(n-2 p+1)}\left(\lambda C_{2}\right)\right\} \\
& +2 k_{o}^{2 p} \lambda^{p}\left\{H_{2(n-p+1)}\left(\lambda C_{1}\right)-H_{2(n-p+1)}\left(\lambda C_{2}\right)\right\}+2\left(\lambda k_{o}\right)^{2 p}\left\{H_{2(n-p+1)}\left(\lambda C_{1}\right)-H_{2(n-p+1)}\left(\lambda C_{2}\right)\right\} \\
& -2 \lambda^{p} k_{o}^{2 p}\left\{H_{2 n+2}\left(\lambda C_{1}\right)-H_{2 n+2}\left(\lambda C_{2}\right)\right\}
\end{aligned}
$$

Thus the MSE can be simplified to be

$$
\begin{aligned}
\operatorname{MSE}\left(\hat{k}_{U}^{p P T}\right)=\left(\lambda k_{o}\right)^{2 p} & {\left[\frac{\Gamma(n-2 p+1) \Gamma(n+1)}{\Gamma^{2}(n-p+1)}-1\right]-\left(\lambda k_{o}\right)^{2 p} \frac{\Gamma(n-2 p+1) \Gamma(n+1)}{\Gamma^{2}(n-p+1)}\left\{H_{2(n-2 p+1)}\left(\lambda C_{1}\right)-H_{2(n-2 p+1)}\left(\lambda C_{2}\right)\right\} } \\
& +\left(1-2 \lambda^{p}\right) k_{o}^{2 p}\left\{H_{2 n+2}\left(\lambda C_{1}\right)-H_{2 n+2}\left(\lambda C_{2}\right)\right\} \\
& +2\left(\lambda k_{o}\right)^{2 p}\left\{H_{2(n-p+1)}\left(\lambda C_{1}\right)-H_{2(n-p+1)}\left(\lambda C_{2}\right)\right\}
\end{aligned}
$$

Next, the bias and MSE of PTE of $k^{p}$ based on MLE are

$$
\begin{gathered}
\operatorname{Bias}\left(\hat{k}_{M L}^{p}{ }^{P T}\right)=\left(\lambda k_{o}(n+1)\right)^{p} \frac{\Gamma(n-p+1)}{\Gamma(n+1)}\left[1-\left\{H_{2(n-p+1)}\left(\lambda C_{1}\right)-H_{2(n-p+1)}\left(\lambda C_{2}\right)\right\}\right]+k_{o}^{p}\left\{H_{2 n+2}\left(\lambda C_{1}\right)-H_{2 n+2}\left(\lambda C_{2}\right)\right\} \\
-\left(\lambda k_{o}\right)^{p}
\end{gathered}
$$

and

$$
\begin{aligned}
& M S E\left(\hat{k}_{M L}^{p P T}\right)=\left\{\left(\lambda k_{o}(n+1)\right)^{p} \frac{\Gamma(n-p+1)}{\Gamma(n+1)}\right\}^{2}\left[\frac{\Gamma(n-2 p+1) \Gamma(n+1)}{\Gamma^{2}(n-p+1)}-1\right] \\
&-\left(\lambda k_{o}(n+1)^{2 p} \frac{\Gamma(n-2 p+1)}{\Gamma(n+1)}\left\{H_{2(n-2 p+1)}\left(\lambda C_{1}\right)-H_{2(n+2 p+1)}\left(\lambda C_{2}\right)\right\}\right. \\
&-\left\{\left(\lambda k_{o}(n+1)\right)^{p} \frac{\Gamma(n-p+1)}{\Gamma(n+1)}\right\}^{2}\left\{H_{2(n-p+1)}\left(\lambda C_{1}\right)-H_{2(n-p+1)}\left(\lambda C_{2}\right)\right\}^{2} \\
&+k_{o}^{2 p}\left\{H_{2 n+2}\left(\lambda C_{1}\right)-H_{2 n+2}\left(\lambda C_{2}\right)\right\}\left[1-\left\{H_{2 n+2}\left(\lambda C_{1}\right)-H_{2 n+2}\left(\lambda C_{2}\right)\right\}\right] \\
&+2\left\{\left(\lambda k_{o}(n+1)\right)^{p} \frac{\Gamma(n-p+1)}{\Gamma(n+1)}\right\}^{2}\left\{H_{2(n-p+1)}\left(\lambda C_{1}\right)-H_{2(n-p+1)}\left(\lambda C_{2}\right)\right\} \\
&+2 k_{o}^{2 p}(\lambda(n+1))^{p} \frac{\Gamma(n-p+1)}{\Gamma(n+1)}\left\{H_{2 n+2}\left(\lambda C_{1}\right)-H_{2 n+2}\left(\lambda C_{2}\right)\right\}\left[\left\{H_{2(n-p+1)}\left(\lambda C_{1}\right)-H_{2(n-p+1)}\left(\lambda C_{2}\right)\right\}-1\right] \\
&+\left[\left(\lambda k_{o}(n+1)\right)^{p} \frac{\Gamma(n-p+1)}{\Gamma(n+1)}\left[1-\left\{H_{2(n-p+1)}\left(\lambda C_{1}\right)-H_{2(n-p+1)}\left(\lambda C_{2}\right)\right\}\right]+k_{o}^{p}\left\{H_{2 n+2}\left(\lambda C_{1}\right)-H_{2 n+2}\left(\lambda C_{2}\right)\right\}\right. \\
&\left.-\left(\lambda k_{o}\right)^{p}\right]^{2}
\end{aligned}
$$


Before deriving bias and MSE for PTE based on EBE, for the sake of simplicity we define the following:

$\varphi_{1}=\int_{0}^{\infty}\left(y+\hat{\mu}_{M L}\right)^{-p} y^{n} e^{-k y} d y, \varphi_{2}=\int_{0}^{\infty}\left(y+\hat{\mu}_{M L}\right)^{-2 p} y^{n} e^{-k y} d y, \varphi_{3}=\int_{\frac{C_{2}}{2 k_{o}}}^{\frac{C_{1}}{2 k_{o}}}\left(y+\hat{\mu}_{M L}\right)^{-p} y^{n} e^{-k y} d y$ and $\varphi_{4}=\int_{\frac{C_{2}}{2 k_{o}}}^{\frac{C_{1}}{2 k_{o}}}\left(y+\hat{\mu}_{M L}\right)^{-2 p} y^{n} e^{-k y} d y$

Therefore, we have the bias and MSE of PTE of $k^{p}$ based on EBE as $\operatorname{Bias}\left(\hat{k}_{E B}^{p}{ }^{P T}\right)=\frac{\Gamma\left(n+\hat{v}_{M L}+p+1\right)\left(\lambda k_{o}\right)^{n+1}}{\Gamma\left(n+\hat{v}_{M L}+1\right) \Gamma(n+1)}\left(\varphi_{1}-\varphi_{3}\right)+k_{o}^{p}\left\{H_{2 n+2}\left(\lambda C_{1}\right)-H_{2 n+2}\left(\lambda C_{2}\right)\right\}-\left(\lambda k_{o}\right)^{p}$

and

$$
\begin{aligned}
& M S E\left(\hat{k}_{E B}^{p}{ }^{P T}\right)=\left\{\frac{\Gamma\left(n+\hat{v}_{M L}+p+1\right)}{\Gamma\left(n+\hat{v}_{M L}+1\right)}\right\}^{2} \frac{\left(\lambda k_{o}\right)^{n+1}\left(\varphi_{2}-\varphi_{4}\right)}{\Gamma(n+1)}-\left[\frac{\Gamma\left(n+\hat{v}_{M L}+p+1\right)\left(\lambda k_{o}\right)^{n+1}}{\Gamma\left(n+\hat{v}_{M L}+1\right) \Gamma(n+1)}\right]^{2}\left(\varphi_{1}-\varphi_{3}\right)^{2} \\
&+ k_{o}^{2 p}\left\{H_{2 n+2}\left(\lambda C_{1}\right)-H_{2 n+2}\left(\lambda C_{2}\right)\right\}\left[1-\left\{H_{2 n+2}\left(\lambda C_{1}\right)-H_{2 n+2}\left(\lambda C_{2}\right)\right\}\right] \\
&+2 k_{o}^{p} \frac{\Gamma\left(n+\hat{v}_{M L}+p+1\right)\left(\lambda k_{o}\right)^{n+1}}{\Gamma\left(n+\hat{v}_{M L}+1\right) \Gamma(n+1)}\left(\varphi_{3}-\varphi_{1}\right)\left\{H_{2 n+2}\left(\lambda C_{1}\right)-H_{2 n+2}\left(\lambda C_{2}\right)\right\} \\
&+\left[\frac{\Gamma\left(n+\hat{v}_{M L}+p+1\right)\left(\lambda k_{o}\right)^{n+1}}{\Gamma\left(n+\hat{v}_{M L}+1\right) \Gamma(n+1)}\left(\varphi_{1}-\varphi_{3}\right)+k_{o}^{p}\left\{H_{2 n+2}\left(\lambda C_{1}\right)-H_{2 n+2}\left(\lambda C_{2}\right)\right\}\right. \\
&\left.-\left(\lambda k_{o}\right)^{p}\right]^{2}
\end{aligned}
$$

Next, the bias and MSE of PTE of $k^{p}$ based on MRE are

$$
\begin{gathered}
\operatorname{Bias}\left(\hat{k}_{M R}^{P T}\right)=\frac{(n-1) \lambda k_{o}}{n}\left[1-\left\{H_{2 n}\left(\lambda C_{1}\right)-H_{2 n}\left(\lambda C_{2}\right)\right\}\right]+k_{o}\left\{H_{2 n+2}\left(\lambda C_{1}\right)-H_{2 n+2}\left(\lambda C_{2}\right)\right\} \\
-\lambda k_{o}
\end{gathered}
$$

and

$$
\begin{aligned}
& \operatorname{MSE}\left(\hat{k}_{M R}^{P T}\right)=\frac{\left(\lambda k_{o}\right)^{2}(n-1)}{n^{2}}-\frac{\left(\lambda k_{o}\right)^{2}(n-1)}{n}\left\{H_{2 n-2}\left(\lambda C_{1}\right)-H_{2 n-2}\left(\lambda C_{2}\right)\right\} \\
&+k_{o}^{2}\left\{H_{2 n+2}\left(\lambda C_{1}\right)-H_{2 n+2}\left(\lambda C_{2}\right)\right\}\left[1-\left\{H_{2 n+2}\left(\lambda C_{1}\right)-H_{2 n+2}\left(\lambda C_{2}\right)\right\}\right]-\frac{\left(\lambda k_{o}\right)^{2}(n-1)^{2}}{n^{2}}\left\{H_{2 n}\left(\lambda C_{1}\right)-H_{2 n}\left(\lambda C_{2}\right)\right\}^{2} \\
&+\frac{2 \lambda k_{o}^{2}(n-1)}{n}\left\{H_{2 n+2}\left(\lambda C_{1}\right)-H_{2 n+2}\left(\lambda C_{2}\right)\right\}\left[1+\left\{H_{2 n}\left(\lambda C_{1}\right)-H_{2 n}\left(\lambda C_{2}\right)\right\}\right] \\
&-\frac{2\left(\lambda k_{o}\right)^{2}(n-1)^{2}}{n^{2}}\left\{H_{2 n}\left(\lambda C_{1}\right)-H_{2 n}\left(\lambda C_{2}\right)\right\} \\
&+\left[\frac{(n-1) \lambda k_{o}}{n}\left[1-\left\{H_{2 n}\left(\lambda C_{1}\right)-H_{2 n}\left(\lambda C_{2}\right)\right\}\right]+k_{o}\left\{H_{2 n+2}\left(\lambda C_{1}\right)-H_{2 n+2}\left(\lambda C_{2}\right)\right\}\right. \\
&\left.-\lambda k_{o}\right]^{2}
\end{aligned}
$$

Now, we derive bias and MSE expressions of PTES of $R(t)$ based on MLE and UMVUE. For sake of simplicity, we define the following:

$$
\varphi_{5}=\int_{C_{2} / 2}^{C_{1}} \frac{z^{n}}{n !} \exp \left(-\left(z+\frac{k(n+1) U(t)}{z}\right)\right) d z \text { and } \varphi_{6}=\int_{C_{2} / 2}^{C_{1} / 2} \frac{z^{n}}{n !} \exp \left(-\left(z+\frac{2 k(n+1) U(t)}{z}\right)\right) d z
$$

Then the bias of PTE of $R(t)$ based on MLE can be derived as follows. We have $\operatorname{Bias}\left(\hat{R}(t)^{P T}\right)=\frac{1}{\Gamma(n+1)} \int_{0}^{\infty} \exp \left[-\left\{y+\frac{k(n+1) U(t)}{y}\right\}\right] y^{n} d y-\varphi_{5}+R_{o}(t)\left\{H_{2 n+2}\left(\lambda C_{1}\right)-H_{2 n+2}\left(\lambda C_{2}\right)\right\}-R(t)$ 
Applying a result of Watson (1952) given by $\int_{0}^{\infty} u^{-m} \exp \left\{-\left(a u+\frac{b}{u}\right)\right\} d u=2\left(\frac{a}{b}\right)^{\frac{m-1}{2}} K_{m-1}(2 \sqrt{a b})$ [it is to be noted that $K_{-m}(\cdot)=K_{m}(\cdot)$ for $m=0,1,2, \ldots$ ], we get $\operatorname{Bias}\left(\hat{R}(t)^{P T}\right)=\frac{2}{n !}\{k(n+1) U(t)\}^{\frac{n+1}{2}} K_{n+1}(2 \sqrt{k(n+1) U(t)})-\varphi_{5}+R_{o}(t)\left\{H_{2 n+2}\left(\lambda C_{1}\right)-H_{2 n+2}\left(\lambda C_{2}\right)\right\}$
$-R(t)$

Following which the MSE of PTE of $R(t)$ based on MLE is obtained as

$$
\begin{aligned}
\operatorname{MSE}\left(\hat{R}(t)^{P T}\right)=\frac{2}{n !}\{ & k(n+1) U(t)\}^{\frac{n+1}{2}} K_{n+1}(2 \sqrt{2 k(n+1) U(t)})-\left[\frac{2}{n !}\{k(n+1) U(t)\}^{\frac{n+1}{2}} K_{n+1}(2 \sqrt{k(n+1) U(t)})\right]^{2}-\varphi_{6}-\varphi_{5}{ }^{2} \\
& +\left(R_{o}(t)\right)^{2}\left\{H_{2 n+2}\left(\lambda C_{1}\right)-H_{2 n+2}\left(\lambda C_{2}\right)\right\}\left\{1-\left\{H_{2 n+2}\left(\lambda C_{1}\right)-H_{2 n+2}\left(\lambda C_{2}\right)\right\}\right\} \\
& +\frac{4}{n !}\{k(n+1) U(t)\}^{\frac{n+1}{2}} K_{n+1}(2 \sqrt{k(n+1) U(t)}) \varphi_{5} \\
& +2 R_{o}(t)\left\{H_{2 n+2}\left(\lambda C_{1}\right)-H_{2 n+2}\left(\lambda C_{2}\right)\right\}\left(\varphi_{5}-\frac{2}{n !}\{k(n+1) U(t)\}^{\frac{n+1}{2}} K_{n+1}(2 \sqrt{k(n+1) U(t)})\right) \\
+ & {\left[\frac{2}{n !}\{k(n+1) U(t)\}^{\frac{n+1}{2}} K_{n+1}(2 \sqrt{k(n+1) U(t)})-\varphi_{5}+R_{o}(t)\left\{H_{2 n+2}\left(\lambda C_{1}\right)-H_{2 n+2}\left(\lambda C_{2}\right)\right\}\right.} \\
& -R(t)]^{2}
\end{aligned}
$$

Denoting by

$$
\varphi_{7}=\int_{C_{2}}^{C_{1}}\left(1-\frac{2 k U(t)}{u}\right)^{n} \frac{u^{n} e^{\frac{-u}{2}}}{2^{n+1} n !} d u \quad \text { and } \varphi_{8}=\int_{C_{2}}^{C_{1}}\left(1-\frac{2 k U(t)}{u}\right)^{2 n} \frac{u^{n} e^{\frac{-u}{2}}}{2^{n+1} n !} d u
$$

The bias of $R(t)$ based on UMVUE can be obtained as follows. We have $\operatorname{Bias}\left(\tilde{R}(t)^{P T}\right)=R_{o}(t)\left\{H_{2 n+2}\left(\lambda C_{1}\right)-H_{2 n+2}\left(\lambda C_{2}\right)\right\}-\varphi_{7}$

From Chaturvedi and Malhotra (2017),

$$
\begin{aligned}
\operatorname{Var}\{\tilde{R}(t)\}=\frac{1}{n !}\{k U(t)\}^{(n+1)} \exp \{-k U(t)\}\left[\frac{a_{n}}{k U(t)}-a_{n-1} \exp \{k U(t)\} E_{i}(-k U(t))\right. \\
+\sum_{i=0}^{n-2} a_{i}\left\{\sum_{m=1}^{n-i-1} \frac{(m-1) !}{(n-i-1) !}(-k U(t))^{n-i-m-1}-\frac{1}{(n-i-1) !}(-k U(t))^{n-i-1} \exp (k U(t)) E_{i}(-k U(t))\right\} \\
\left.+\sum_{i=n+1}^{2 n} a_{i}(i-n) !\left(\frac{1}{k U(t)}\right) \sum_{r=0}^{i-n+1} \frac{1}{r !}(k U(t))^{r}\right]-\exp \{-2 k U(t)\}
\end{aligned}
$$

where $a_{i}=(-1)^{i}\left(\begin{array}{c}2 n \\ i\end{array}\right)$ and $-E_{i}(-x)=\int_{x}^{\infty} \frac{e^{-u}}{u} d u$.

Thus the MSE of $R(t)$ based on UMVUE is

$$
\begin{aligned}
\operatorname{MSE}\left(\tilde{R}(t)^{P T}\right)=\frac{1}{n !}\{ & k U(t)\}^{(n+1)} \exp \{-k U(t)\}\left[\frac{a_{n}}{k U(t)}-a_{n-1} \exp \{k U(t)\} E_{i}(-k U(t))\right. \\
& +\sum_{i=0}^{n-2} a_{i}\left\{\sum_{m=1}^{n-i-1} \frac{(m-1) !}{(n-i-1) !}(-k U(t))^{n-i-m-1}-\frac{1}{(n-i-1) !}(-k U(t))^{n-i-1} \exp (k U(t)) E_{i}(-k U(t))\right\} \\
& \left.+\sum_{i=n+1}^{2 n} a_{i}(i-n) !\left(\frac{1}{k U(t)}\right) \sum_{r=0}^{i-n+1} \frac{1}{r !}(k U(t))^{r}\right]-\exp \{-2 k U(t)\}-\varphi_{8}+\left(R_{o}(t)\right)^{2}\left\{H_{2 n+2}\left(\lambda C_{1}\right)-H_{2 n+2}\left(\lambda C_{2}\right)\right\} \\
& +2 \varphi_{7} R(t)-2 R(t) R_{o}(t)\left\{H_{2 n+2}\left(\lambda C_{1}\right)-H_{2 n+2}\left(\lambda C_{2}\right)\right\}
\end{aligned}
$$

Now, we derive bias and MSE expressions of PTES of $P$ based on MLE. We have $\operatorname{Bias}\left(\hat{P}^{P T}\right)=E(\hat{P})-E(\hat{P} I(B))+P_{o} E(I(B))-P$ 
where $E(I(B))=P(B)=P\left(C_{4}<F_{2(n+1), 2(m+1)}<C_{3}\right)$ and $E(\widehat{P})=E\left(\frac{\widehat{k_{2}}}{\widehat{k_{1}}+\widehat{k_{2}}}\right)=E(\hat{Q})$, (say). Following the approach by Constantine et al. (1986), we obtain the $p d f$ of $\hat{Q}$ by transformation into two new independent $r v s \quad r>0$ and $\beta \in\left(0, \frac{\pi}{2}\right)$ where $\widehat{k_{1}}=k_{1} r(n+1) \cos ^{2} \beta$ and $\widehat{k_{2}}=k_{2} r(m+$ 1) $\sin ^{2} \beta$. Putting $\varphi=\cos ^{2} \beta$, the $p d f$ of $\hat{Q}=\left[1+\rho\left(\frac{n+1}{m+1}\right)\left(\frac{\varphi}{1-\varphi}\right)\right]^{-1}$ is

$g(q)=\frac{1}{\beta(n+1, m+1)}\left(\rho\left(\frac{n+1}{m+1}\right)\right)^{n+1} \frac{q^{n}(1-q)^{m}}{(1+\varepsilon q)^{n+m+2}} ; 0<q<1, \varepsilon=\rho\left(\frac{n+1}{m+1}\right)-1$

When $\varepsilon=0,(2.33)$ gives

$$
E\left(\hat{Q}^{l}\right)=\frac{\beta(n+l+1, m+1)}{\beta(n+1, m+1)}
$$

When $\varepsilon \neq 0$, (2.33) yields on substituting $1+\varepsilon q=t$,

$$
E\left(\hat{Q}^{l}\right)=\frac{1}{\beta(n+1, m+1)}\left(\rho\left(\frac{n+1}{m+1}\right)\right)^{n+1} \frac{1}{\varepsilon^{n+m+l-1}} \int_{1}^{1+\varepsilon}(t-1)^{n+l}(1+\varepsilon-t)^{m} t^{-(n+m+2)} d t
$$

Thus, for $\varphi_{10}=\frac{1}{\beta(n+1, m+1)}\left(\rho\left(\frac{n+1}{m+1}\right)\right)^{n+1} \frac{1}{\varepsilon^{n+m}} \int_{1}^{1+\varepsilon}(t-1)^{n+1}(1+\varepsilon-t)^{m} t^{-(n+m+2)} d t$

$$
E(\widehat{P})=\left\{\begin{array}{c}
\frac{n+1}{n+m+2} ; \varepsilon=0 \\
\varphi_{10} ; \varepsilon \neq 0
\end{array}\right.
$$

Also, $E(\hat{P} I(B))=\int_{C_{4}}^{C_{3}}\left(\frac{1}{1+\frac{k_{1}}{k_{2} F}}\right) \Phi_{1}(F) d F=\varphi_{9}$ where $\Phi_{1}(\cdot)$ is the $p d f$ of $F-$ distribution with $(2(n+1), 2(m+1))$ degrees of freedom. Then the bias and MSE of PTE of $P$ based on MLE are obtained as $\operatorname{Bias}\left(\hat{P}^{P T}\right)=\left\{\begin{array}{r}P_{o} P(B)-\varphi_{9} ; \varepsilon=0 \\ \varphi_{10}-\varphi_{9}+P_{o} P(B)-P ; \varepsilon \neq 0\end{array}\right.$

and $\operatorname{MSE}\left(\hat{P}^{P T}\right)=\left\{\begin{array}{l}\left(\frac{n+1}{n+m+2}\right)^{2}\left[\left(\frac{n+2}{n+1}\right)\left(\frac{n+m+2}{n+m+3}\right)-1\right]-\varphi_{11}+2\left(\frac{n+1}{n+m+2}\right)\left(\varphi_{9}-P_{o} P(B)\right)+P_{o}^{2} P(B) ; \varepsilon=0 \\ \varphi_{12}-\varphi_{11}+P_{o}^{2} P(B)+\left(\frac{n+1}{n+m+2}\right)^{2}+2\left(\frac{n+1}{n+m+2}\right)\left(\varphi_{9}-\varphi_{10}\right)-2\left(\frac{n+1}{n+m+2}\right) P_{o} P(B) ; \varepsilon \neq 0\end{array}\right.$ where $\varphi_{11}=\int_{C_{4}}^{C_{3}}\left(\frac{1}{1+\frac{k_{1}}{k_{2} F}}\right)^{2} \Phi_{1}(F) d F$ and $\varphi_{12}=\frac{1}{\beta(n+1, m+1)}\left(\rho\left(\frac{n+1}{m+1}\right)\right)^{n+1} \frac{1}{\varepsilon^{n+m+1}} \int_{1}^{1+\varepsilon}(t-1)^{n+2}(1+\varepsilon-$ $t)^{m} t^{-(n+m+2)} d t$

Finally, we obtain the bias of PTE of $P$ based on UMVUE as $\operatorname{Bias}\left(\tilde{P}^{P T}\right)=\left\{\begin{array}{l}P_{O} P(B)-\varphi_{13} ; v \leq 1 \\ P_{o} P(B)-\varphi_{14} ; v>1\end{array}\right.$ where $v=\frac{U\left(R_{n}\right)}{U\left(R_{m}^{*}\right)}, \varphi_{13}=\sum_{i=0}^{m-1} \frac{(-1)^{i} m ! n !}{(m-i-1) !(n+i+1) ! \beta(n+1, m+1)}\left(\frac{k_{2}}{k_{1}}\right)^{i+1} \int_{C_{4}^{\prime}}^{C_{3}^{\prime}} \frac{z^{n+i+1}}{(1+z)^{n+m+2}} d z$ and $\varphi_{14}=\sum_{i=0}^{n} \frac{(-1)^{i} n ! m !}{(n-i) !(m+i) ! \beta(n+1, m+1)}\left(\frac{k_{1}}{k_{2}}\right)^{i} \int_{C_{4}^{\prime}}^{C_{3}^{\prime}} \frac{z^{n-i}}{(1+z)^{n+m+2}} d z, C_{3}^{\prime}=\left(\frac{n+1}{m+1}\right) C_{3}$ and $C_{4}^{\prime}=\left(\frac{n+1}{m+1}\right) C_{4}$.

To obtain the MSE of PTE of $P$ based on UMVUE, consider $E\left(\tilde{P}^{2}\right)=E\left(\sum_{i=0}^{m-1} \sum_{j=0}^{m-1} a_{i} a_{j}(v)^{i+j+2} \mid v \leq 1\right) P(v \leq 1)+E\left(\sum_{i=0}^{n} \sum_{j=0}^{n} b_{i} b_{j}(v)^{-(i+j)} \mid v>1\right) P(v>1)$ 
where, $a_{i}=\frac{(-1)^{i} m ! n !}{(m-i-1) !(n+i+1) !}, b_{i}=\frac{(-1)^{i} n ! m !}{(n-i) !(m+i) !}$. An explicit expression of $\operatorname{Var}(\tilde{P})$ depends on the evaluation of $E\left(v^{l} \mid v \leq 1\right) P(v \leq 1)$ and $E\left(v^{-l} \mid v>1\right) P(v>1)$ for $l \geq 0$. To evaluate them we first obtain the $p d f$ of $v$. We have, $v=\frac{U\left(R_{n}\right)}{U\left(R_{m}^{*}\right)}$ which implies, $\frac{(m+1) k_{1}}{(n+1) k_{2}} v=\rho \frac{(m+1)}{(n+1)} v \sim F_{2(n+1), 2(m+1)}$. Thus we obtain the $p d f$ of $v$ as

$$
h(v)=\frac{\rho^{n+1}}{\beta(n+1, m+1)} v^{n}(1+\rho v)^{-n-m-2} ; v>0
$$

For $l>0$,

$$
E\left(v^{l} \mid v \leq 1\right) P(v \leq 1)=\int_{0}^{1} \frac{\rho^{n+1}}{\beta(n+1, m+1)} v^{n+l}(1+\rho v)^{-n-m-2} d v .
$$

Substituting $r=(1+\rho v)^{-1}$, the binomial expansion of the integrand yields,

$$
E\left(v^{l} \mid v \leq 1\right) P(v \leq 1)=\frac{\rho^{-l}}{\beta(n+1, m+1)} \sum_{i=0}^{n+l}(-1)^{i}\left(\begin{array}{c}
n+l \\
i
\end{array}\right) \int_{\rho^{\prime}}^{1} r^{m-l+i} d r
$$

where $\int_{\rho^{\prime}}^{1} r^{m-l+i} d r=\left\{\begin{array}{c}\frac{1-\left(\rho^{\prime}\right)^{m-l+i+1}}{m-l+k+1} ; i \neq l-m-1 \\ -\log \left(\rho^{\prime}\right) ; i=l-m-1\end{array}\right.$ and $\rho^{\prime}=\frac{1}{1+\rho}$. Similarly we can obtain,

$$
E\left(v^{-l} \mid v>1\right) P(v>1)=\frac{\rho^{l}}{\beta(n+1, m+1)} \sum_{i=0}^{m+l}(-1)^{i}\left(\begin{array}{c}
m+l \\
i
\end{array}\right) \int_{1-\rho^{\prime}}^{1} r^{n-l+i} d r
$$

where $\int_{1-\rho^{\prime}}^{1} r^{n-l+i} d r=\left\{\begin{array}{c}\frac{1-\left(1-\rho^{\prime}\right)^{n-l+i+1}}{n-l+k+1} ; i \neq l-n-1 \\ -\log \left(1-\rho^{\prime}\right) ; i=l-n-1\end{array}\right.$. Thus, $\operatorname{Var}(\tilde{P})=\varphi_{15}-P^{2}$, where

$$
\begin{aligned}
\varphi_{15}=\sum_{i=0}^{m-1} \sum_{j=0}^{m-1} \frac{a_{i} a_{j} \rho^{-(i+j+2)}}{\beta(n+1, m+1)} \sum_{p=0}^{n+i+j+2}(-1)^{p}\left(\begin{array}{c}
n+i+j+2 \\
p
\end{array}\right) \int_{\rho^{\prime}}^{1} r^{m+p-i-j-2} d r \\
+\sum_{i=0}^{n} \sum_{j=0}^{n} \frac{b_{i} b_{j} \rho^{i+j}}{\beta(n+1, m+1)} \sum_{p=0}^{m+i+j}(-1)^{p}\left(\begin{array}{c}
m+i+j \\
p
\end{array}\right) \int_{1-\rho^{\prime}}^{1} r^{n+p-i-j} d r
\end{aligned}
$$

and $\operatorname{Var}(\tilde{P} I(B))=\left\{\begin{array}{l}\varphi_{16}-\varphi_{13}^{2} ; v \leq 1 \\ \varphi_{17}-\varphi_{14}^{2} ; v>1\end{array}\right.$, where $\varphi_{16}=\sum_{i=0}^{m-1} \sum_{j=0}^{m-1} a_{i} a_{j} \int_{C_{4}}^{C_{3}} v^{i+j+2} \Phi_{1}(F) d F$ and $\varphi_{17}=$ $\sum_{i=0}^{n} \sum_{j=0}^{n} b_{i} b_{j}\left(\frac{\theta_{2}(m+1)}{\theta_{1}(n+1)}\right)^{i+j} \int_{C_{4}}^{C_{3}} v^{i+j} \Phi_{1}(F) d F$. Thus the MSE of PTE of $P$ based on UMVUE is obtained as

$\operatorname{MSE}\left(\tilde{P}^{P T}\right)=\left\{\begin{array}{l}\varphi_{15}-P^{2}-\varphi_{16}+2 P\left(\varphi_{13}-P_{o} P(B)\right)+P_{o}^{2} P(B) ; v \leq 1 \\ \varphi_{15}-P^{2}-\varphi_{17}+2 P\left(\varphi_{14}-P_{o} P(B)\right)+P_{o}^{2} P(B) ; v>1\end{array}\right.$

Comparing the performance of the proposed PTES analytically is a complicated task because of their formulations. Therefore several figures as well as some numerical results are presented to discuss their performance. The relative efficiency of PTE of some parameter $\tau$ denoted by $\hat{\tau}_{\delta}^{P T}$ over its regular estimator $\hat{\tau}_{\delta}$ is defined as follows:

$$
e\left(\hat{\tau}_{\delta}^{P T}, \hat{\tau}_{\delta}\right)=\frac{\operatorname{MSE}\left(\hat{\tau}_{\delta}\right)}{\operatorname{MSE}\left(\hat{\tau}_{\delta}^{P T}\right)}
$$

where $\delta \in\{U, M L, M R, E B\}$. 


\subsection{Proposed PTCI for parameter $k$}

In this section, we construct preliminary test confidence interval (PTCI) of the parameter $k$. Suppose for known value of the other parameters $c$ and $\alpha$, we are interested in testing the hypothesis

$$
\begin{aligned}
& H_{o}: k=k_{o} \\
& H_{1}: k \neq k_{o}
\end{aligned}
$$

Since $U\left(R_{n}\right)$ follows gamma distribution with parameters $(n+1, k)$, it is easy to obtain the $100(1-\varepsilon) \%$ equal tail confidence interval (ETCI) of $k$ as

$$
I_{E T C I}=\left[\frac{\chi_{2(n+1)}^{2}\left(\frac{\varepsilon}{2}\right)}{2 U\left(R_{n}\right)}, \frac{\chi_{2(n+1)}^{2}\left(1-\frac{\varepsilon}{2}\right)}{2 U\left(R_{n}\right)}\right]
$$

From equation (2.3), we obtain the MRE of $k$ as $\hat{k}_{M R}=\frac{(n-1)}{U\left(R_{n}\right)}$. Then we can re-write $I_{E T C I}$ based on MRE of $k$ as

$$
I_{M R}^{E T C I}=\left[C_{5} \hat{k}_{M R}, C_{6} \hat{k}_{M R}\right]
$$

where $C_{5}=\frac{\chi_{2(n+1)}^{2}\left(\frac{\varepsilon}{2}\right)}{2(n-1)}$ and $C_{6}=\frac{\chi_{2(n+1)}^{2}\left(1-\frac{\varepsilon}{2}\right)}{2(n-1)}$.

Accordingly, we can define PTCI of $k$ based MRE of $k$ as

$$
I_{M R}^{P T C I}=\left[C_{5} \hat{k}_{M R}^{P T}, C_{6} \hat{k}_{M R}^{P T}\right]
$$

If we let $\lambda=\frac{k}{k_{o}}$ and $T=2 k U\left(R_{n}\right)$, then the coverage probability (CP) of PTCI of $k$ based on MRE of $k$ is defined as

$$
\begin{aligned}
P\left(k \in I_{M R}^{P T C I}\right)= & P\left(k \in\left(c_{5} k_{o}, c_{6} k_{o}\right): \chi_{2(n+1)}^{2}\left(\frac{\varepsilon}{2}\right)<2 k U\left(R_{n}\right)<\chi_{2(n+1)}^{2}\left(1-\frac{\varepsilon}{2}\right)\right)+P\left(k \in\left(c_{5} \hat{k}_{M R}, c_{6} \hat{k}_{M R}\right): 2 k U\left(R_{n}\right)<\chi_{2(n+1)}^{2}\left(\frac{\varepsilon}{2}\right)\right) \\
& +P\left(k \in\left(c_{5} \hat{k}_{M R}, c_{6} \hat{k}_{M R}\right): 2 k U\left(R_{n}\right)>\chi_{2(n+1)}^{2}\left(1-\frac{\varepsilon}{2}\right)\right) \\
= & P\left(\left(c_{5}<\lambda<c_{6}\right): \lambda \chi_{2(n+1)}^{2}\left(\frac{\varepsilon}{2}\right)<T<\lambda \chi_{2(n+1)}^{2}\left(1-\frac{\varepsilon}{2}\right)\right)+P\left(\chi_{2(n+1)}^{2}\left(\frac{\varepsilon}{2}\right)<T<\chi_{2(n+1)}^{2}\left(1-\frac{\varepsilon}{2}\right), T<\lambda \chi_{2(n+1)}^{2}\left(\frac{\varepsilon}{2}\right)\right) \\
& \quad+P\left(\chi_{2(n+1)}^{2}\left(\frac{\varepsilon}{2}\right)<T<\chi_{2(n+1)}^{2}\left(1-\frac{\varepsilon}{2}\right), T>\lambda \chi_{2(n+1)}^{2}\left(1-\frac{\varepsilon}{2}\right)\right) \\
= & P\left(\lambda \chi_{2(n+1)}^{2}\left(\frac{\varepsilon}{2}\right)<T<\lambda \chi_{2(n+1)}^{2}\left(1-\frac{\varepsilon}{2}\right)\right) I_{\left(c_{5}, c_{6}\right)}(\lambda)+P\left(\chi_{2(n+1)}^{2}\left(\frac{\varepsilon}{2}\right)<T<\min \left\{\chi_{2(n+1)}^{2}\left(1-\frac{\varepsilon}{2}\right), \lambda \chi_{2(n+1)}^{2}\left(\frac{\varepsilon}{2}\right)\right\}\right) \\
& \quad+P\left(\max \left\{\chi_{2(n+1)}^{2}\left(\frac{\varepsilon}{2}\right), \lambda \chi_{2(n+1)}^{2}\left(1-\frac{\varepsilon}{2}\right)\right\}<T<\chi_{2(n+1)}^{2}\left(1-\frac{\varepsilon}{2}\right)\right)
\end{aligned}
$$

Denoting the first term of the above equation by $C$, then we obtain the CP of PTCI based on MRE of $k$ as 


$$
P\left(k \in I_{M R}^{P T C I}\right)=\left\{\begin{array}{c}
C+1-\varepsilon ; 0<\lambda \leq \frac{\chi_{(n+1)}^{2}\left(\frac{\varepsilon}{2}\right)}{\chi_{2(n+1)}^{2}\left(1-\frac{\varepsilon}{2}\right)} \text { or } \lambda>\frac{\chi_{2(n+1)}^{2}\left(1-\frac{\varepsilon}{2}\right)}{\chi_{2(n+1)}^{2}\left(\frac{\varepsilon}{2}\right)} \\
C+P\left(\lambda \chi_{2(n+1)}^{2}\left(1-\frac{\varepsilon}{2}\right)<T<\chi_{2(n+1)}^{2}\left(1-\frac{\varepsilon}{2}\right)\right) ; \frac{\chi_{2(n+1)}^{2}\left(\frac{\varepsilon}{2}\right)}{\chi_{2(n+1)}^{2}\left(1-\frac{\varepsilon}{2}\right)}<\lambda \leq 1 \\
C+P\left(\chi_{2(n+1)}^{2}\left(\frac{\varepsilon}{2}\right)<T<\lambda \chi_{2(n+1)}^{2}\left(\frac{\varepsilon}{2}\right)\right) ; 1<\lambda \leq \frac{\chi_{2(n+1)}^{2}\left(1-\frac{\varepsilon}{2}\right)}{\chi_{2(n+1)}^{2}\left(\frac{\varepsilon}{2}\right)}
\end{array}\right.
$$

In order to find the expected length of PTCI of $k$, we first obtain the length of PTCI based on MRE of $k$ which is given by the following $r v$ :

$$
L_{M R}^{P T C I}=\left\{\begin{array}{c}
k_{o}\left(c_{6}-c_{5}\right) ; \chi_{2(n+1)}^{2}\left(\frac{\varepsilon}{2}\right)<2 k_{o} U\left(R_{n}\right)<\chi_{2(n+1)}^{2}\left(1-\frac{\varepsilon}{2}\right) \\
\hat{k}_{M R}\left(c_{6}-c_{5}\right) ; 2 k_{o} U\left(R_{n}\right)<\chi_{2(n+1)}^{2}\left(\frac{\varepsilon}{2}\right) \text { or } 2 k_{o} U\left(R_{n}\right)>\chi_{2(n+1)}^{2}\left(1-\frac{\varepsilon}{2}\right)
\end{array}\right.
$$

Then the expected length (EL) of the PTCI of $k$ based on MRE of $k$ is given by

$$
\begin{gathered}
E\left(L_{M R}^{P T C I}\right)=E\left(L_{M R}^{P T C I} \mid \chi_{2(n+1)}^{2}\left(\frac{\varepsilon}{2}\right)<2 k_{o} U\left(R_{n}\right)<\chi_{2(n+1)}^{2}\left(1-\frac{\varepsilon}{2}\right)\right) P\left(\chi_{2(n+1)}^{2}\left(\frac{\varepsilon}{2}\right)<2 k_{o} U\left(R_{n}\right)<\chi_{2(n+1)}^{2}\left(1-\frac{\varepsilon}{2}\right)\right) \\
+E\left(L_{M R}^{P T C I} \mid 2 k_{o} U\left(R_{n}\right)<\chi_{2(n+1)}^{2}\left(\frac{\varepsilon}{2}\right) \text { or } 2 k_{o} U\left(R_{n}\right)>\chi_{2(n+1)}^{2}\left(1-\frac{\varepsilon}{2}\right)\right) P\left(2 k_{o} U\left(R_{n}\right)<\chi_{2(n+1)}^{2}\left(\frac{\varepsilon}{2}\right) \text { or } 2 k_{o} U\left(R_{n}\right)\right. \\
\left.>\chi_{2(n+1)}^{2}\left(1-\frac{\varepsilon}{2}\right)\right) \\
=k_{o}\left(c_{6}-c_{5}\right)\left[H_{2 n+2}\left(\lambda \chi_{2(n+1)}^{2}\left(1-\frac{\varepsilon}{2}\right)\right)-H_{2 n+2}\left(\lambda \chi_{2(n+1)}^{2}\left(\frac{\varepsilon}{2}\right)\right)+\lambda\left\{H_{2 n-2}\left(\lambda \chi_{2(n+1)}^{2}\left(\frac{\varepsilon}{2}\right)\right)+1-H_{2 n-2}\left(\lambda \chi_{2(n+1)}^{2}\left(1-\frac{\varepsilon}{2}\right)\right)\right\}\right]
\end{gathered}
$$

On similar lines, we can obtain the CP of PTCI of $k$ based on UMVUE as

$$
P\left(k \in I_{U}^{P T C I}\right)=\left\{\begin{array}{c}
D+1-\varepsilon ; 0<\lambda \leq \frac{\chi_{2(n+1)}^{2}\left(\frac{\varepsilon}{2}\right)}{\chi_{2(n+1)}^{2}\left(1-\frac{\varepsilon}{2}\right)} \text { or } \lambda>\frac{\chi_{2(n+1)}^{2}\left(1-\frac{\varepsilon}{2}\right)}{\chi_{2(n+1)}^{2}\left(\frac{\varepsilon}{2}\right)} \\
D+P\left(\lambda \chi_{2(n+1)}^{2}\left(1-\frac{\varepsilon}{2}\right)<T<\chi_{2(n+1)}^{2}\left(1-\frac{\varepsilon}{2}\right)\right) ; \frac{\chi_{2(n+1)}^{2}\left(\frac{\varepsilon}{2}\right)}{\chi_{2(n+1)}^{2}\left(1-\frac{\varepsilon}{2}\right)}<\lambda \leq 1 \\
D+P\left(\chi_{2(n+1)}^{2}\left(\frac{\varepsilon}{2}\right)<T<\lambda \chi_{2(n+1)}^{2}\left(\frac{\varepsilon}{2}\right)\right) ; 1<\lambda \leq \frac{\chi_{2(n+1)}^{2}\left(1-\frac{\varepsilon}{2}\right)}{\chi_{2(n+1)}^{2}\left(\frac{\varepsilon}{2}\right)}
\end{array}\right.
$$

where $D=P\left(\lambda \chi_{2(n+1)}^{2}\left(\frac{\varepsilon}{2}\right)<T<\lambda \chi_{2(n+1)}^{2}\left(1-\frac{\varepsilon}{2}\right)\right) I_{\left(c_{5}, c_{6}\right)}(\lambda)$ and the EL of the PTCI of $k$ based on UMVUE of $k$ as

$$
\begin{aligned}
E\left(L_{U}^{P C C I}\right)=k_{o}\left(c_{6}-c_{5}\right) & {\left[H_{2 n+2}\left(\lambda \chi_{2(n+1)}^{2}\left(1-\frac{\varepsilon}{2}\right)\right)-H_{2 n+2}\left(\lambda \chi_{2(n+1)}^{2}\left(\frac{\varepsilon}{2}\right)\right)\right.} \\
+ & \left.\frac{\lambda n}{n-1}\left\{H_{2 n-2}\left(\lambda \chi_{2(n+1)}^{2}\left(\frac{\varepsilon}{2}\right)\right)+1-H_{2 n-2}\left(\lambda \chi_{2(n+1)}^{2}\left(1-\frac{\varepsilon}{2}\right)\right)\right\}\right]
\end{aligned}
$$

Finally, we obtain the CP of PTCI of $k$ based on MLE of $k$ is defined as

$$
P\left(k \in I_{M L}^{P T C I}\right)=\left\{\begin{array}{c}
E+1-\varepsilon ; 0<\lambda \leq \frac{\chi_{2(n+1)}^{2}\left(\frac{\varepsilon}{2}\right)}{\chi_{2(n+1)}^{2}\left(1-\frac{\varepsilon}{2}\right)} \text { or } \lambda>\frac{\chi_{2(n+1)}^{2}\left(1-\frac{\varepsilon}{2}\right)}{\chi_{2(n+1)}^{2}\left(\frac{\varepsilon}{2}\right)} \\
E+P\left(\lambda \chi_{2(n+1)}^{2}\left(1-\frac{\varepsilon}{2}\right)<T<\chi_{2(n+1)}^{2}\left(1-\frac{\varepsilon}{2}\right)\right) ; \frac{\chi_{2(n+1)}^{2}\left(\frac{\varepsilon}{2}\right)}{\chi_{2(n+1)}^{2}\left(1-\frac{\varepsilon}{2}\right)}<\lambda \leq 1 \\
E+P\left(\chi_{2(n+1)}^{2}\left(\frac{\varepsilon}{2}\right)<T<\lambda \chi_{2(n+1)}^{2}\left(\frac{\varepsilon}{2}\right)\right) ; 1<\lambda \leq \frac{\chi_{2(n+1)}^{2}\left(1-\frac{\varepsilon}{2}\right)}{\chi_{2(n+1)}^{2}\left(\frac{\varepsilon}{2}\right)}
\end{array}\right.
$$

where $E=P\left(\lambda \chi_{2(n+1)}^{2}\left(\frac{\varepsilon}{2}\right)<T<\lambda \chi_{2(n+1)}^{2}\left(1-\frac{\varepsilon}{2}\right)\right) I_{\left(c_{5}, c_{6}\right)}(\lambda)$ and the EL of the PTCI of $k$ based on MLE of $k$ as 


$$
\begin{aligned}
E\left(L_{M L}^{P T C I}\right)=k_{o}\left(c_{6}-c_{5}\right) & {\left[H_{2 n+2}\left(\lambda \chi_{2(n+1)}^{2}\left(1-\frac{\varepsilon}{2}\right)\right)-H_{2 n+2}\left(\lambda \chi_{2(n+1)}^{2}\left(\frac{\varepsilon}{2}\right)\right)\right.} \\
+ & \left.\frac{\lambda(n+1)}{n-1}\left\{H_{2 n-2}\left(\lambda \chi_{2(n+1)}^{2}\left(\frac{\varepsilon}{2}\right)\right)+1-H_{2 n-2}\left(\lambda \chi_{2(n+1)}^{2}\left(1-\frac{\varepsilon}{2}\right)\right)\right\}\right]
\end{aligned}
$$

\subsection{Numerical Findings}

From the previous section, it is easy to obtain the relative efficiency of PTE over the usual estimator of $k$ based on UMVUE, MLE and MRE denoted by $e\left(\hat{k}_{U}^{P T}, \hat{k}_{U}\right), e\left(\hat{k}_{M L}^{P T}, \hat{k}_{M L}\right)$ and $e\left(\hat{k}_{M R}^{P T}, \hat{k}_{M R}\right)$ respectively, which depend on the sample size $(n+1)$ and level of significance $\varepsilon$. Figure 1 shows the relative efficiency of $\hat{k}_{M L}^{P T}$ over $\hat{k}_{M L}$ and we observe that there exists an interval of $\lambda$ for which this efficiency is greater than 1 . Similarly, Figure 2 and Figure 3 show the relative efficiency of $\hat{k}_{U}^{P T}$ over $\hat{k}_{U}$ and $\hat{k}_{M R}^{P T}$ over $\hat{k}_{M R}$ respectively. Since $e\left(\hat{k}_{E B}^{P T}, \hat{k}_{E B}\right)$ does not have a closed form and thus we use Monte Carlo simulation technique to compute this efficiency based on the following algorithm:

i. For given values of $\mu$ and $v$, generate one sample from $\operatorname{Gamma}(\mu, v)$ and denote it as $k^{*}$.

ii. For a specified value of $n$, generate $m$ random samples from $\operatorname{gamma}\left(n+1, k^{*}\right)$ to obtain $Y_{j} ; j=1,2, \ldots, m$.

iii. Compute, $\hat{k}_{E B}^{p}(j)=\frac{\Gamma(n+v+p+1)}{\Gamma(n+v+1)}\left(Y_{j}+\mu\right)^{p} ; j=1,2, \ldots, m$.

iv. For a specified value of $k_{o}$, test the hypothesis $H_{o}: k=k_{o}$, using the test statistic in equation (2.8) to get $\hat{k}_{E B}^{p{ }^{P T}}(j)=\hat{k}_{E B}^{p}(j)-\left(\hat{k}_{E B}^{p}(j)-k_{o}^{p}\right) I(A) ; j=1,2, \ldots, m$.

v. Compute $\operatorname{MSE}=\frac{1}{m} \sum_{j=1}^{m}\left(\delta(j)-k^{*}\right)^{2}$, where $\delta(j) \in\left\{\hat{k}_{E B}^{p}(j), \hat{k}_{E B}^{p{ }^{P T}}(j)\right\}, \quad j=$ $1,2, \ldots, m$. For $\mu=2, v=4$ and $m=1000$, the result is shown in Figure 4 . 

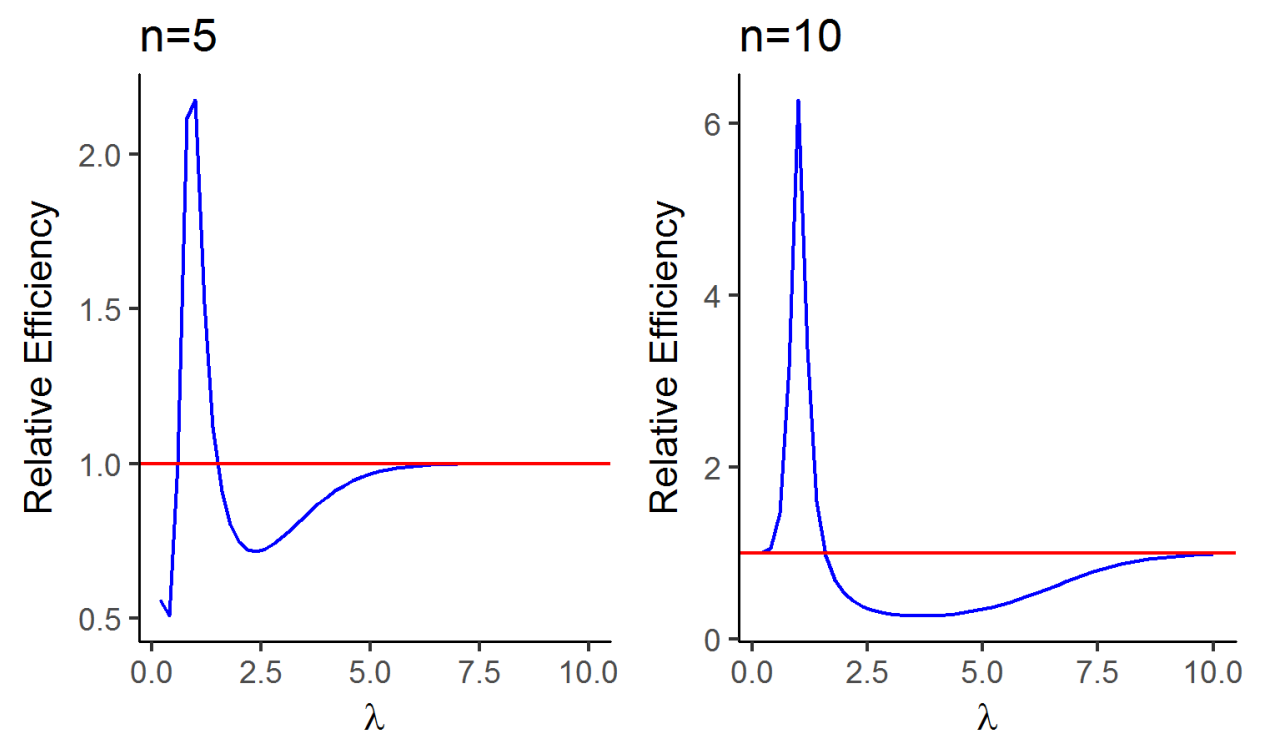

Figure 1: Relative Efficiency of PTE of $k$ based on MLE with respect to $\lambda=\frac{k}{k_{o}}$
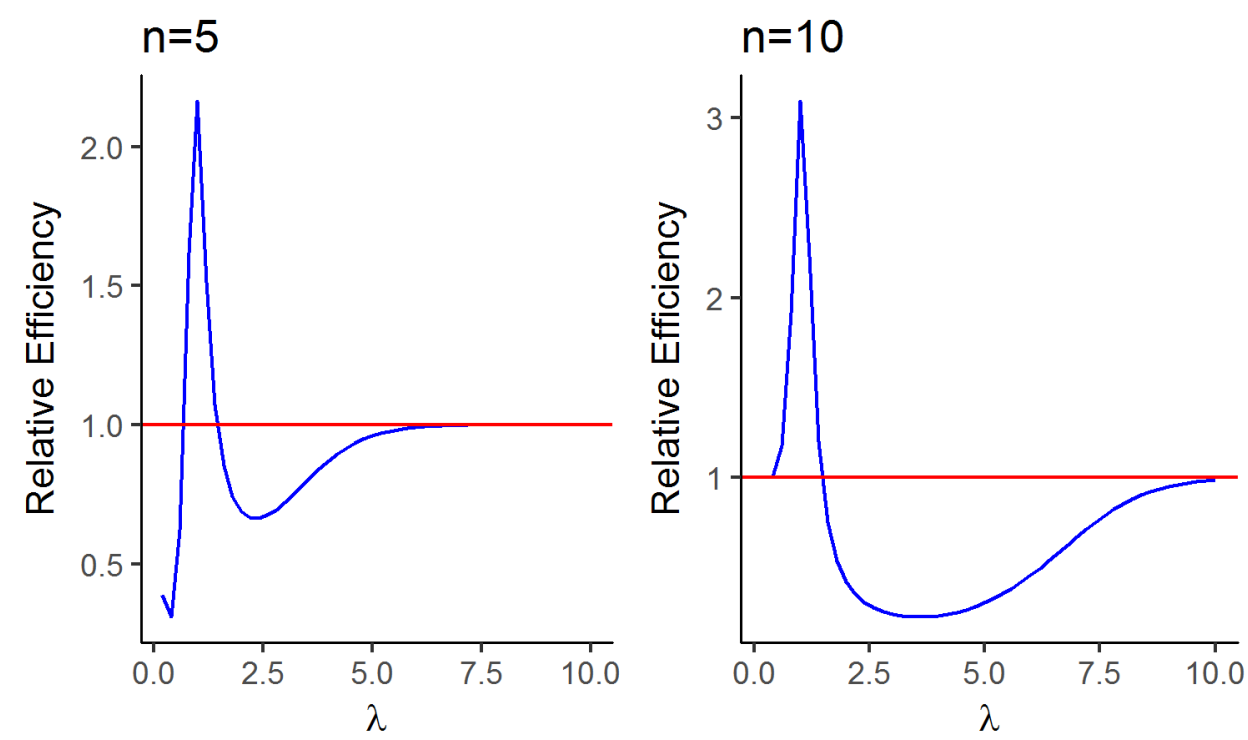

Figure 2: Relative Efficiency of PTE of $k$ based on UMVUE with respect to $\lambda=\frac{k}{k_{o}}$ 

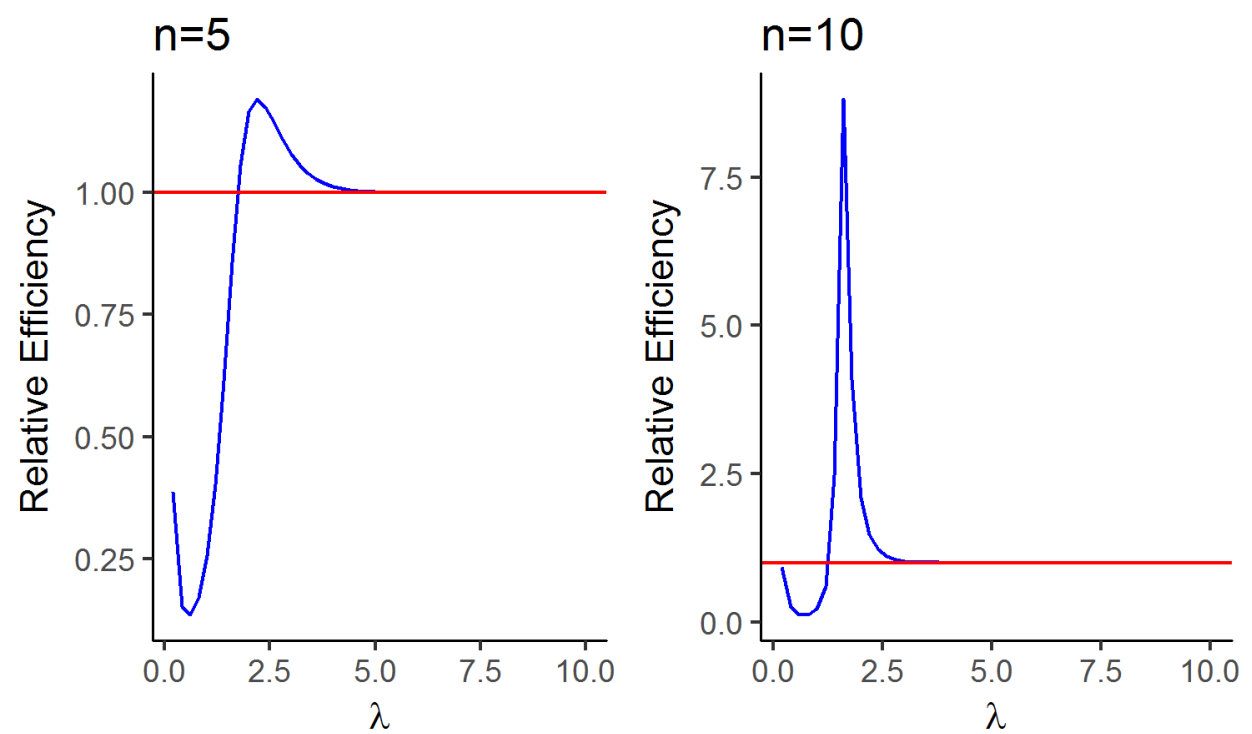

Figure 3: Relative Efficiency of PTE of $k$ based on best invariant estimator with respect to

$$
\lambda=\frac{k}{k_{o}}
$$
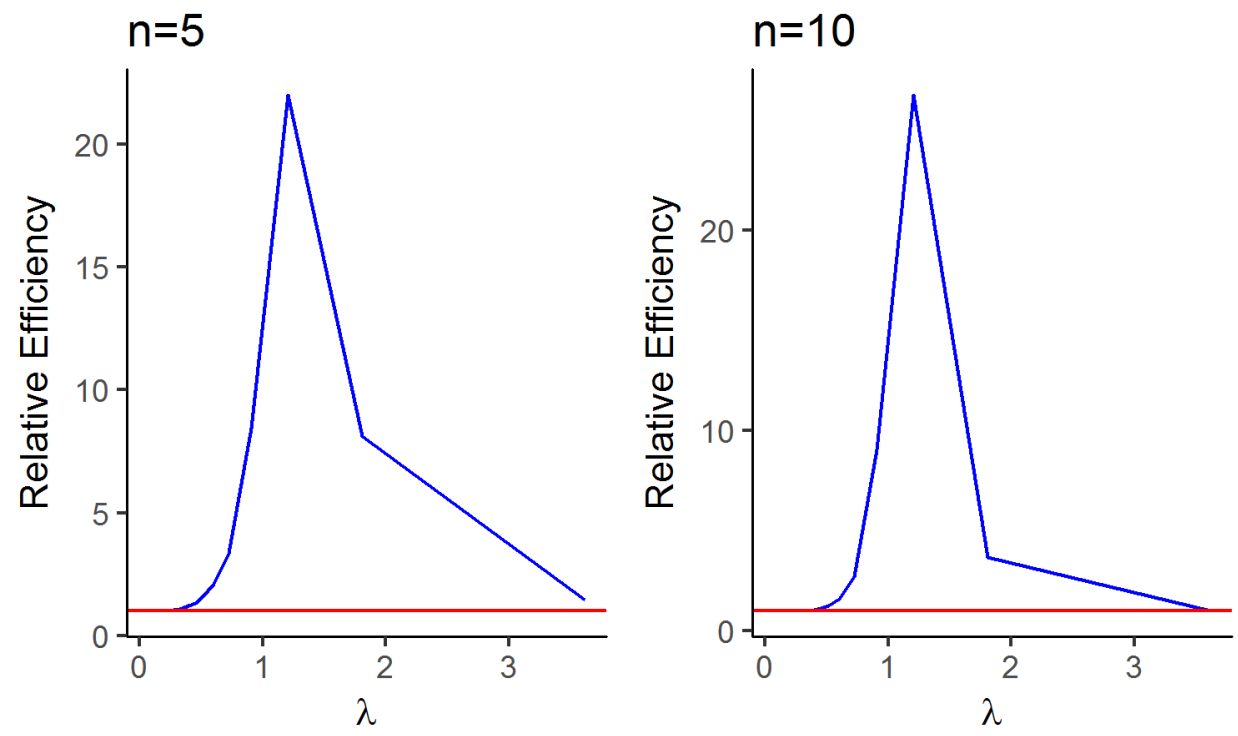

Figure 4: Relative Efficiency of PTE of $k$ based on EBE with respect to $\lambda=\frac{k}{k_{o}}$

From Figures 1, 2, 3 and 4 we observe that as sample size increases, the relative efficiency of PTES of $k$ with respect to $\lambda$ increases in the interval of $\lambda$ for which this efficiency is greater than 1 .

In Figure 5, we compare the performance of the two PTES of $k$ based on UMVUE and MRE of $k$. Even though the MRE of $k$ is biased for $k$, its corresponding PTE is more efficient that the PTE of $k$ based on UMVUE for higher values of $\lambda$. However, in the neighbourhood of the null hypothesis $H_{o}: k=k_{o}$, the PTE of $k$ based on UMVUE continues to be a better estimator. 

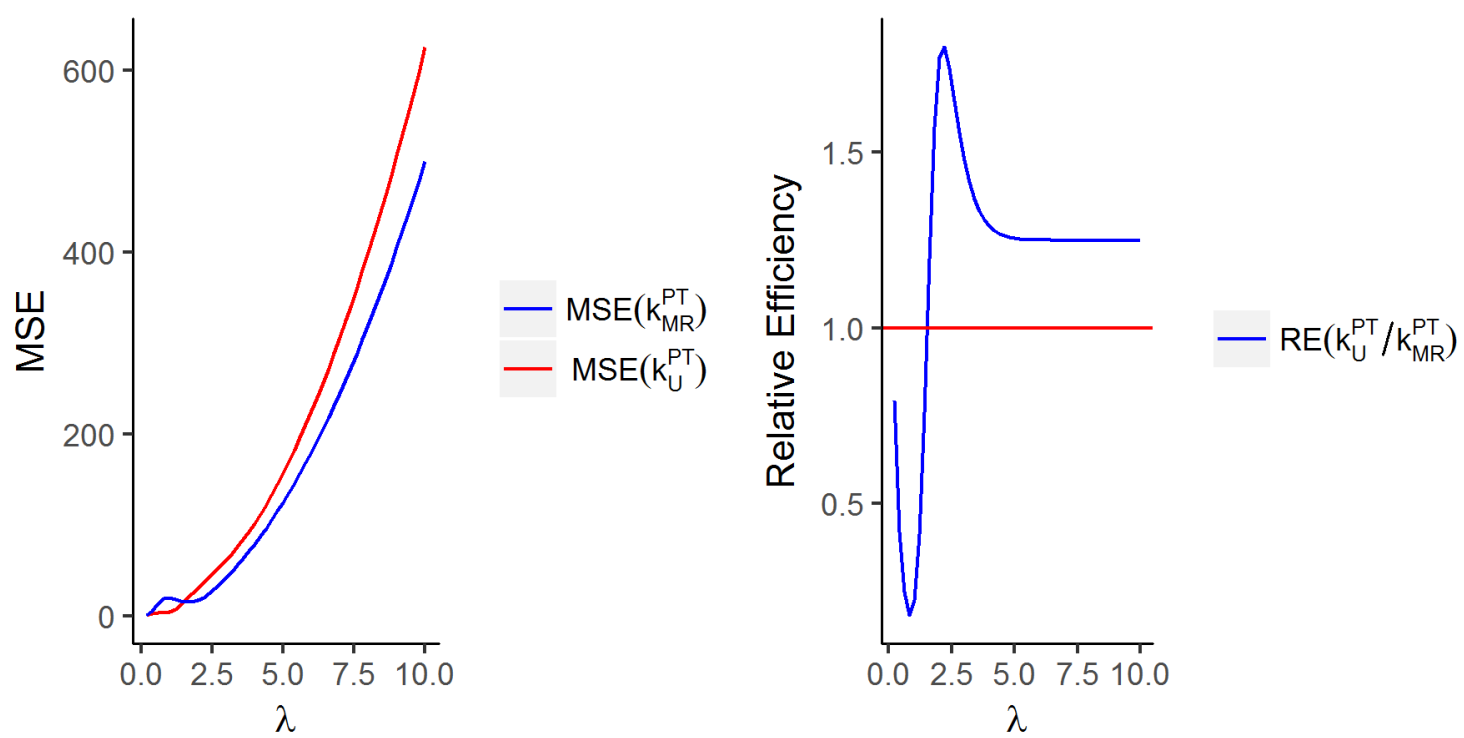

Figure 5: MSE and Relative Efficiency of PTE of $k$ based on UMVUE and EBE with respect

$$
\text { to } \lambda=\frac{k}{k_{o}}
$$

For a fixed sample size $n=5$, Figure 6 compares the relative efficiency of $\hat{R}(t)^{P T}$ over $\hat{R}(t)$ and $\tilde{R}(t)^{P T}$ over $\tilde{R}(t)$ respectively with respect to $\theta=\frac{R(t)}{R_{o}(t)}$ for any fixed time point and level of significance 0.05. From this figure, it is clear that PTES of $R(t)$ based on MLE and UMVUE outperform the usual estimators of $R(t)$ in a particular interval of $\theta$.
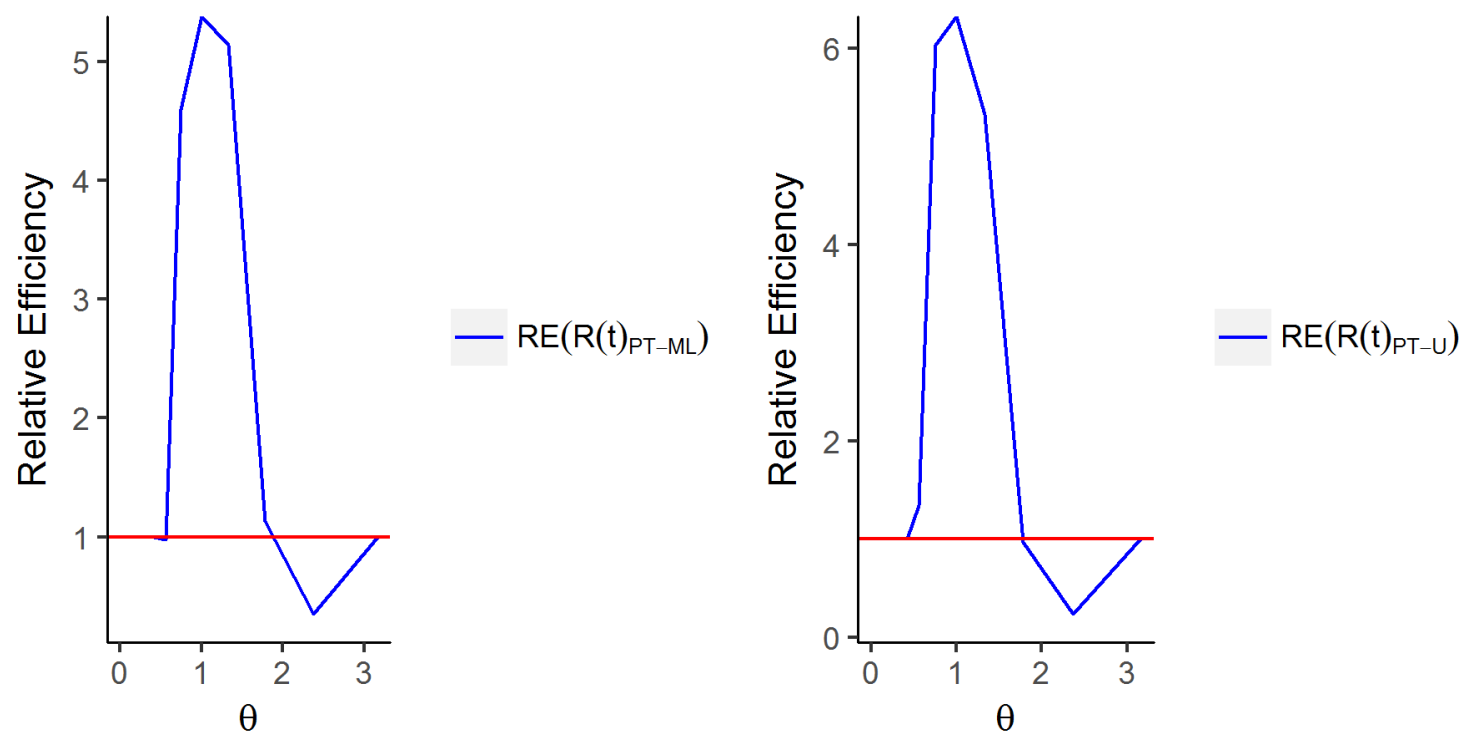

Figure 6: Relative Efficiency of PTE of $R(t)$ based on MLE and UMVUE with respect to

$$
\theta=\frac{R(t)}{R_{o}(t)}
$$

Now we study the relative efficiency of PTES of $P$ based on MLE and UMVUE over the usual estimators of $P$. Suppose for different values of $k_{1}$ and $k_{2}$ we want to test the 
hypothesis $H_{o}: P=P_{o}$ against $H_{1}: P \neq P_{o}$ for fixed sample sizes $n=5$ and $m=2$. Then in Figure 7, the relative efficiency of PTES of $P$ based on MLE and UMVUE over the usual estimators of $P$ has been demonstrated. From this figure, it is clear that PTES of $P$ based on MLE and UMVUE outperform the usual estimators of $P$ in an interval of $\theta=\frac{P}{P_{o}}$.
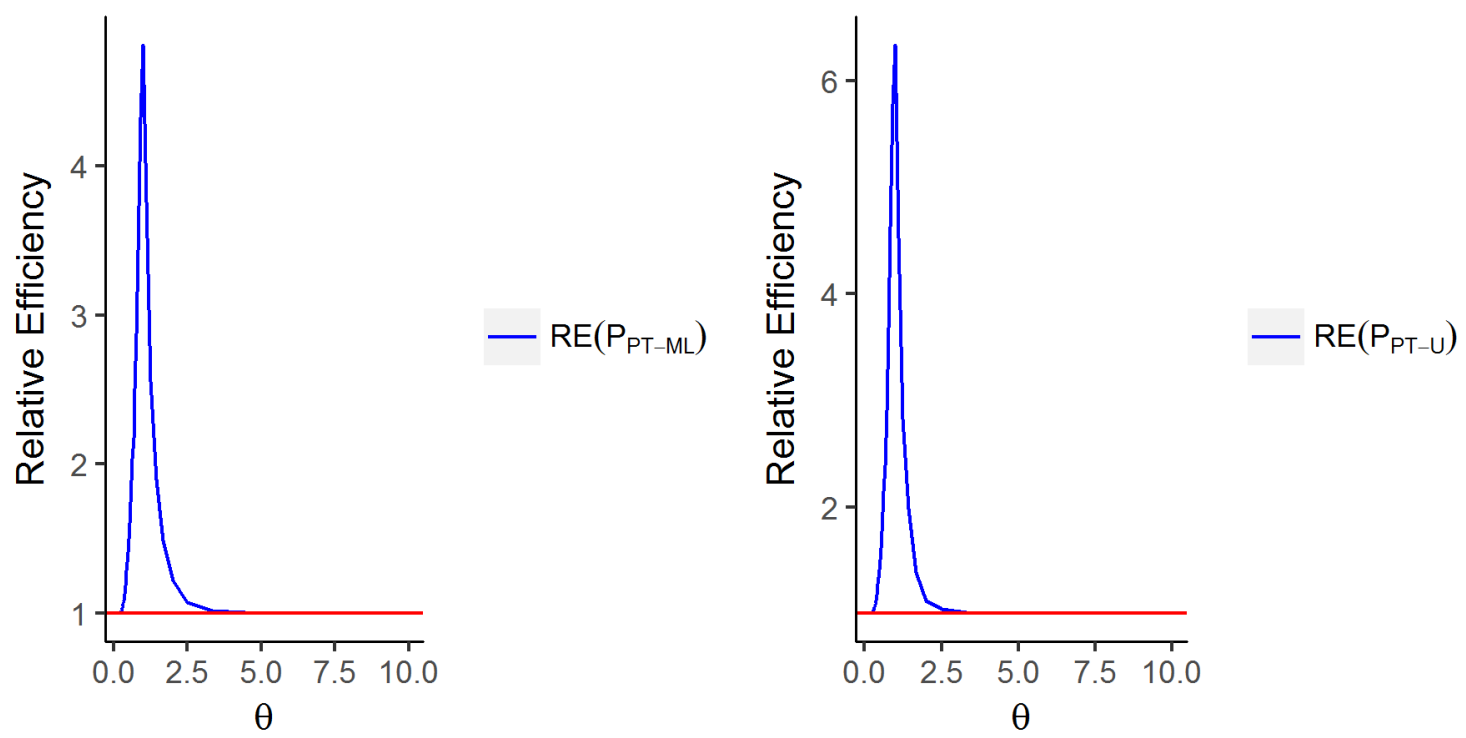

Figure 7: Relative Efficiency of PTE of $P$ based on MLE and UMVUE with respect to $\theta=$

$$
\frac{P}{P_{o}}
$$

In Figure 8, we show the CP of PTCI of $k$ based on MRE with respect to $\lambda=\frac{k}{k_{o}}$ for a fixed sample size and level of significance $\varepsilon$. From the figure and the derived expression of the CP of PTCI, we observe that as the value of $\lambda$ tends to 0 or $\infty$, the CP of PTCI tends to $1-\varepsilon$ and for an interval of $\lambda$ around 1 , the CP of PTCI is greater than $1-\varepsilon$. This domination interval is larger for smaller sample sizes. Thus, we can conclude that the $\mathrm{CP}$ of PTCI of $k$ is greater than the CP of ETCI for some values of $\lambda$ in a specific interval around 1. Similar result has been observed for the CP of PTES of $k$ based on UMVUE and MLE in Figure 9 and Figure 10 respectively. 

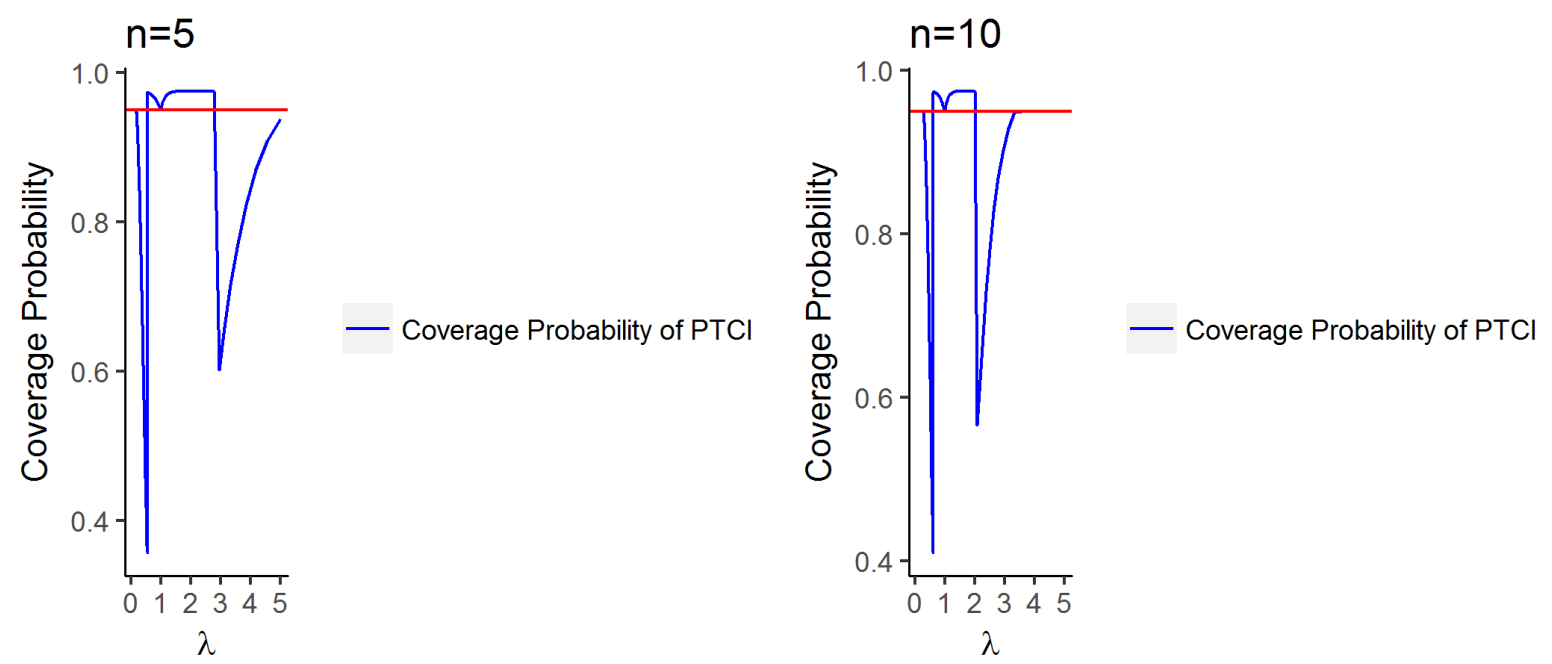

Figure 8: Coverage Probability of PTCI of $k$ based on MRE
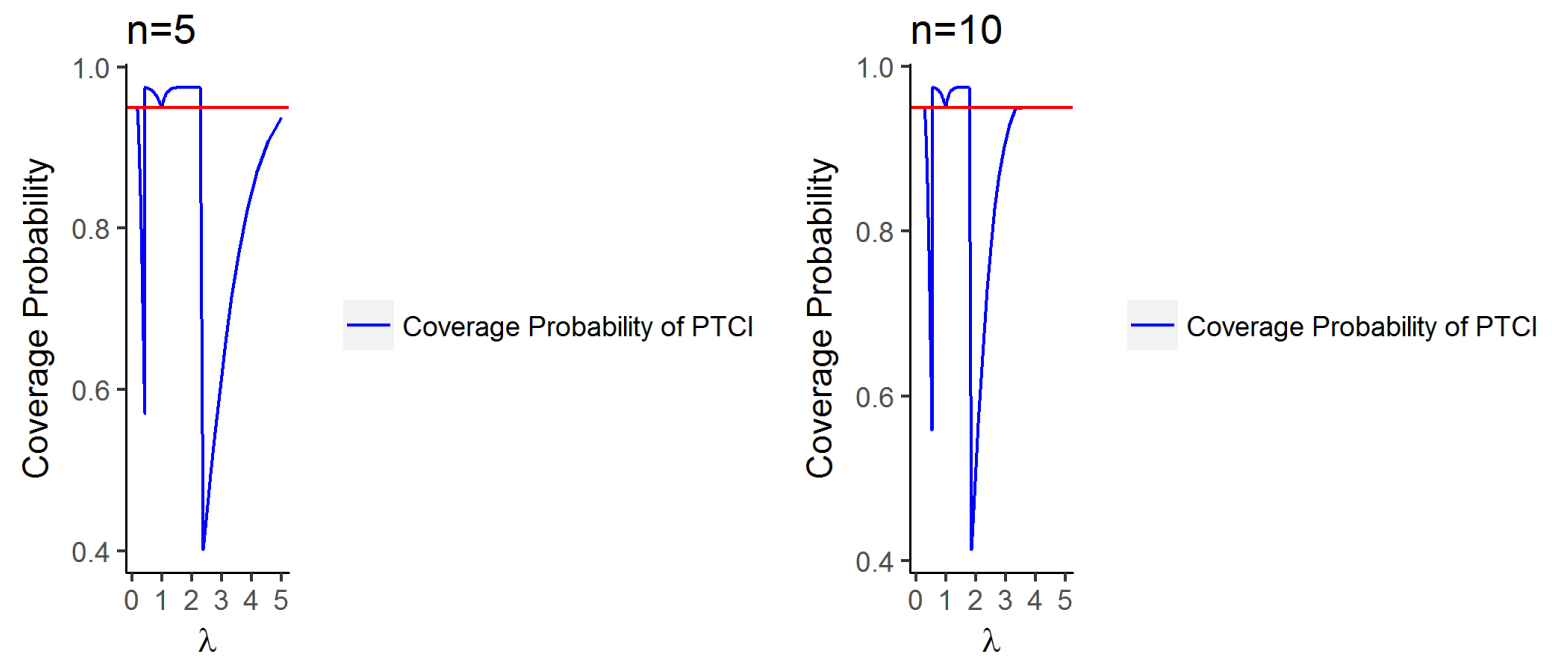

Figure 9: Coverage Probability of PTCI of $k$ based on UMVUE
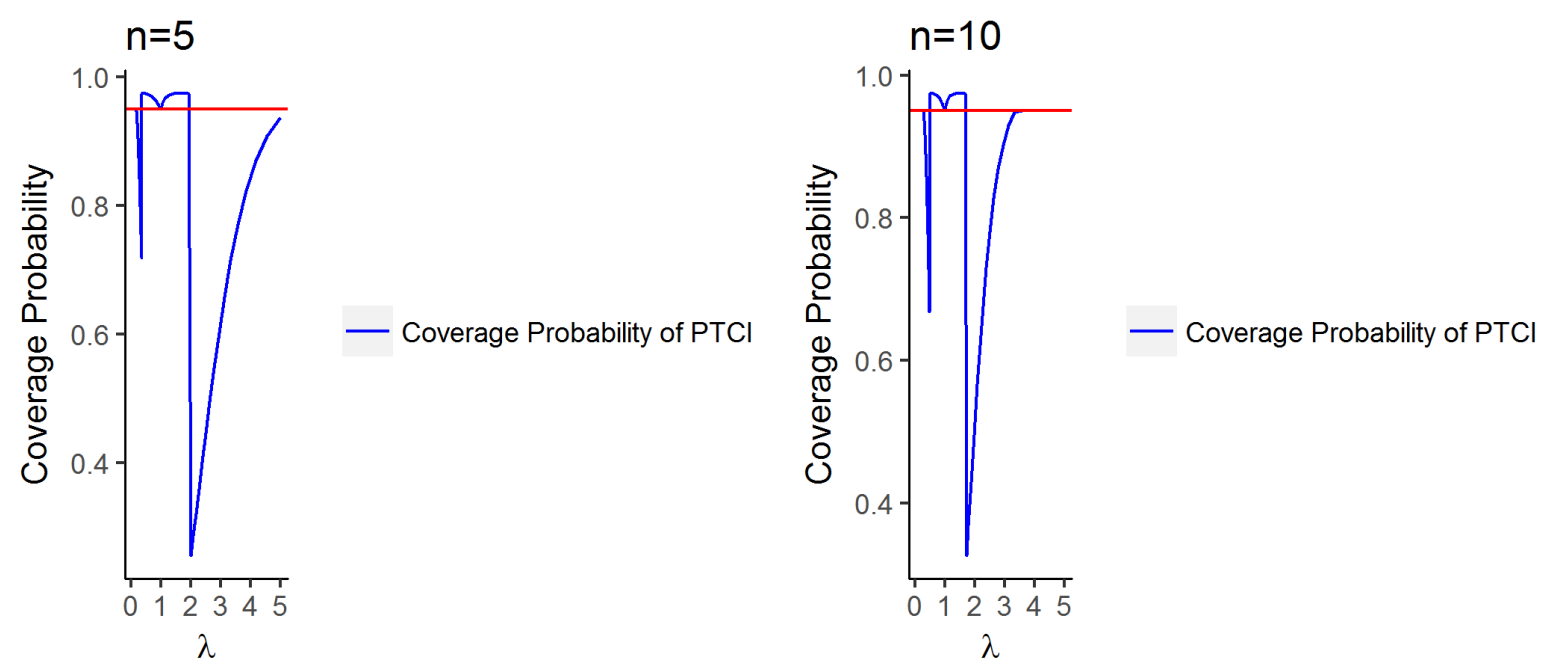

Figure 10: Coverage Probability of PTCI of $k$ based on MLE 
In Figure 11, we compare the scaled EL of PTCI of $k$ based on MRE with the ETCI with respect to $\lambda$. We observe from this figure that there exists an interval of $\lambda$ for which the EL of PTCI is lower than that of ETCI. This interval of $\lambda$ for which EL of PTCI is lower decreases with an increase in sample size. We also note that as $\lambda$ tends to 0 or $\infty$, the EL of PTCI tend to be close to the EL of ETCI. Similar results are observed for EL of PTCI of $k$ based on UMVUE and MLE in Figure 12 and Figure 13 respectively.
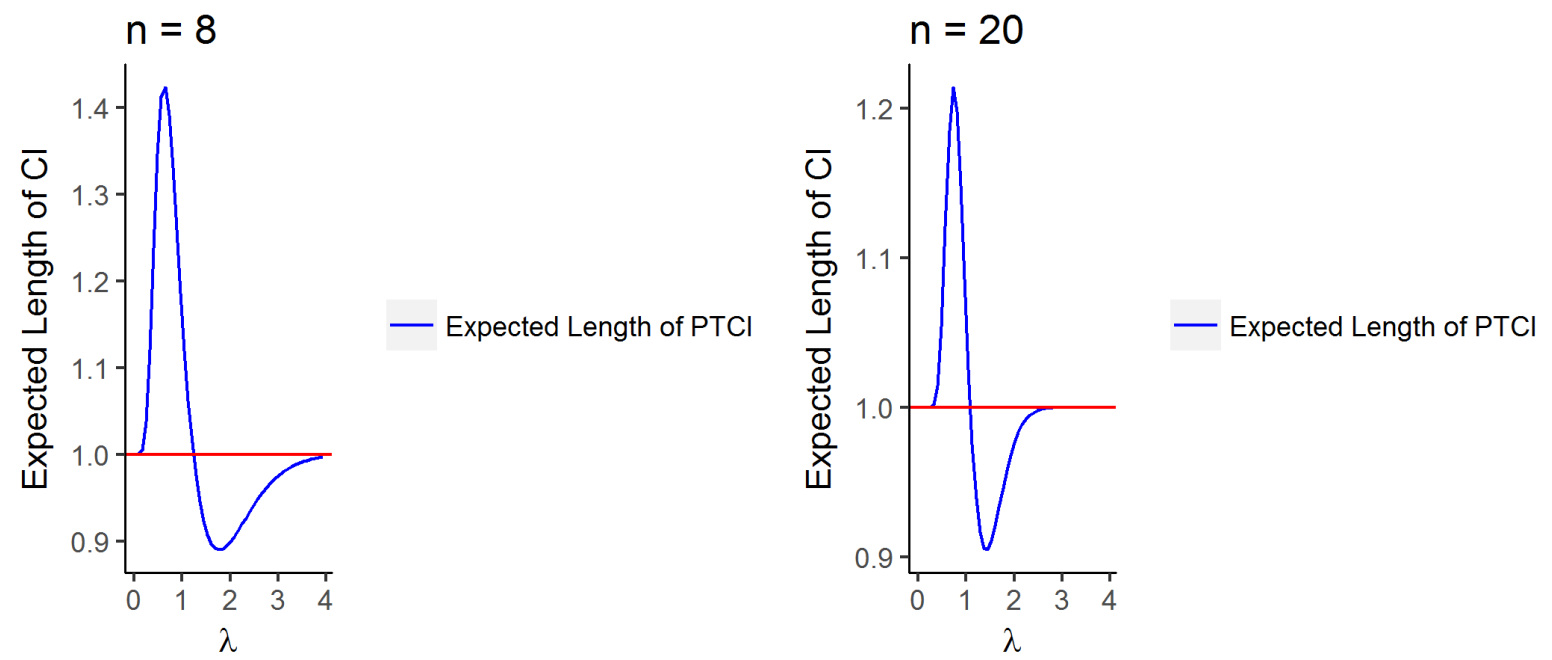

Figure 11: Expected Length of PTCI of $k$ based on MRE
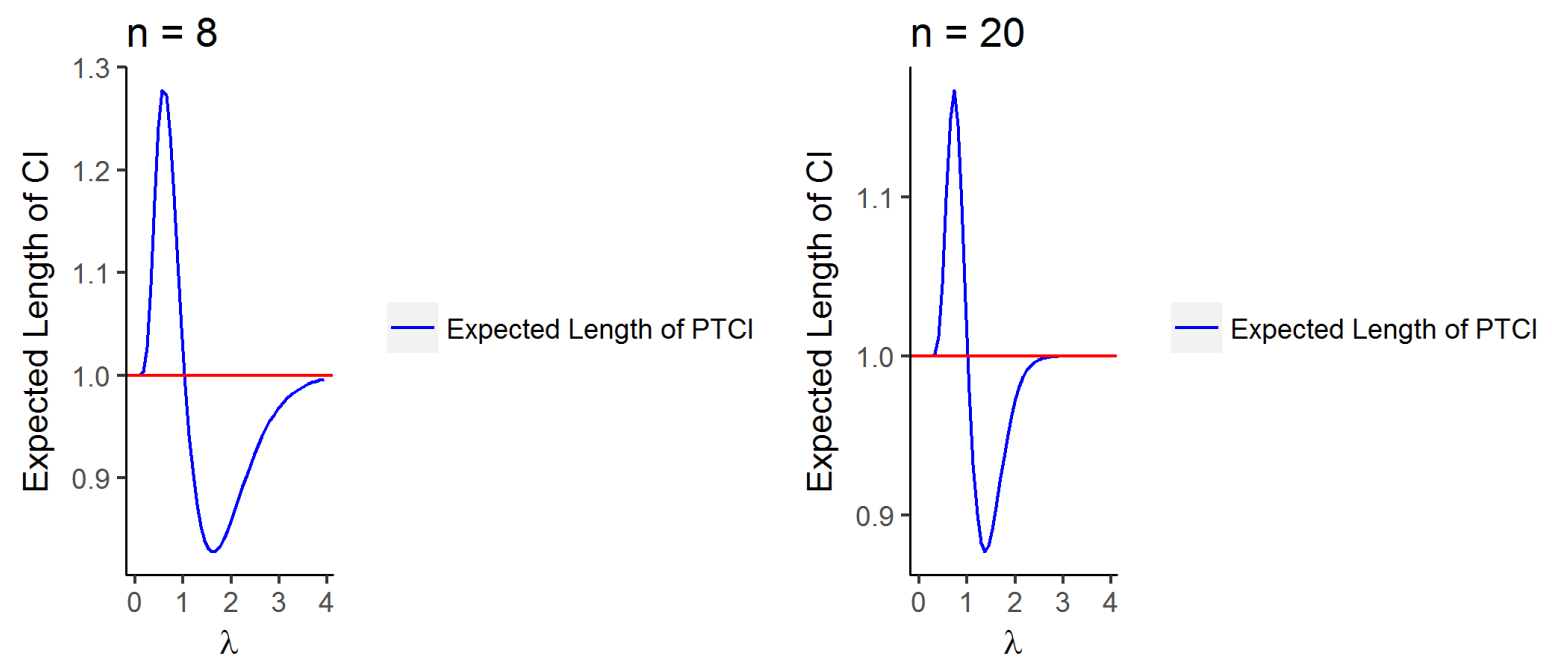

Figure 12: Expected Length of PTCI of $k$ based on UMVUE 

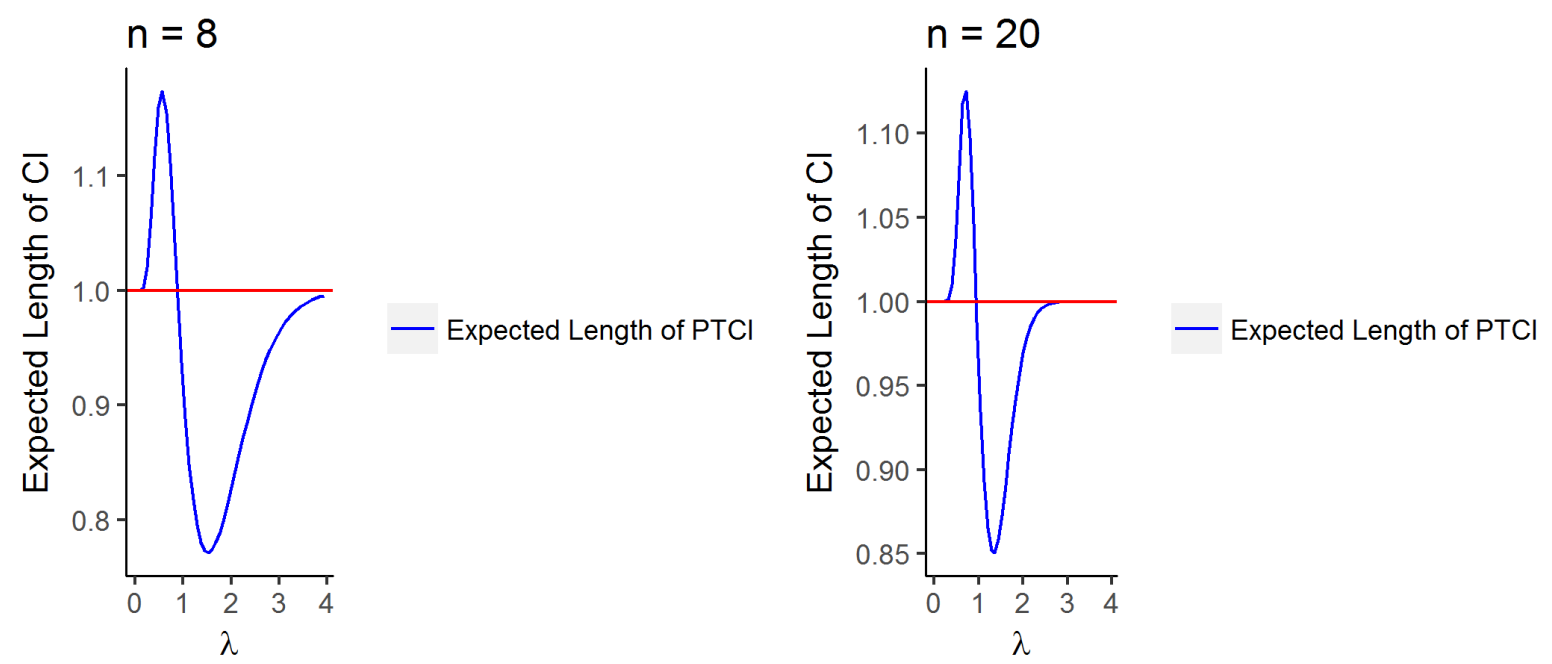

Figure 13: Expected Length of PTCI of $k$ based on MLE

\section{Proposed PTES when all the parameters are unknown}

In this section, we consider the case when all the parameters $k, c$ and $\alpha$ are unknown. Chaturvedi and Malhotra (2017) discussed numerical techniques to obtain the MLES of the parameters $k, c$ and $\alpha$ as $\hat{k}, \hat{c}$ and $\hat{\alpha}$ respectively. They also derived the elements of the observed Fisher information matrix. Since the exact distribution of the test statistics cannot be achieved, so we follow an approach by Gulati and Padgett $(1991,1994,1995)$. Suppose we have $m$ independent samples of size $n+1$ from upper record values like $R_{j 0}, R_{j 1}, \ldots, R_{j n} ; j=1,2, \ldots, m$. Then the MLE of the parameter $k^{p}$ is given by

$\hat{k}_{R}^{p}=\left(\frac{m(n+1)}{\sum_{j=1}^{m} \log \left(1+\frac{R_{j n}^{\hat{c}}}{\hat{\alpha}}\right)}\right)^{p}$

Further, if there exists some prior information on the parameter in the form of $k=k_{o}$ and we are interested to estimate $k$ incorporating such information. So we consider the following simple hypothesis to check the validity of this information:

$$
\begin{aligned}
& H_{o}: k=k_{o} \\
& H_{1}: k \neq k_{o}
\end{aligned}
$$

Under $H_{o}, \frac{\sqrt{m}\left(\hat{k}_{R}-k\right)}{\sqrt{\operatorname{Var}\left(\hat{k}_{R}\right)}} \stackrel{\text { asymp }}{\longrightarrow} N(0,1)$ and the test statistic is defined as

$L_{m-k}=\left(\frac{\sqrt{m}\left(\hat{k}_{R}-k_{o}\right)}{\sqrt{\operatorname{Var}\left(\hat{k}_{R}\right)}}\right)^{2}$ 
Under $H_{o}, L_{m-k}$ converges to central $\chi^{2}$ distribution with 1 degree of freedom as $m \rightarrow$ $\infty$ while under the local alternative of the form

$H_{m}: k=k_{o}+\frac{\delta_{k}}{\sqrt{m}}$

$L_{m}-k$ converges to non-central $\chi^{2}$ distribution with non-centrality parameter

$$
\Delta_{k}^{2}=\left(\frac{\sqrt{m}\left(k-k_{o}\right)}{\sqrt{\operatorname{Var}\left(k_{R}\right)}}\right)^{2}
$$

Based on the asymptotic distribution of $L_{m-k}$, the critical region is given by $L_{m-k}>\chi_{1}^{2}(\varepsilon)$ where $\varepsilon$ is the level of significance. Thus, we define PTE of $k$ as

$\hat{k}_{R}^{p^{P T}}=\hat{k}_{R}^{p}-\left(\hat{k}_{R}^{p}-k_{o}^{p}\right) I\left(L_{m-k}<\chi_{1}^{2}(\varepsilon)\right)$

Note that PTE of the other parameters $c$ and $\alpha$ can also be defined in similar fashion.

Next, by invariance property of MLE, the MLE of the reliability function $R(t)$ under this approach is given by

$\hat{R}(t)_{R}=\exp \left(-\hat{k}_{R} \log \left(1+\frac{t^{\hat{c}}}{\widehat{\alpha}}\right)\right)$

Further if we suspect that $R(t)=R_{o}$ and consider the following simple hypothesis to check the validity of this information:

$$
\begin{aligned}
& H_{o}: R(t)=R_{o} \\
& H_{1}: R(t) \neq R_{o}
\end{aligned}
$$

Under $H_{O}, \frac{\sqrt{m}\left(\hat{R}(t)_{R}-R_{O}\right)}{\sqrt{\operatorname{Var}\left(\hat{R}(t)_{R}\right)}} \stackrel{\text { asymp }}{\longrightarrow} N(0,1)$ and the test statistic is defined as

$L_{m-R(t)}=\left(\frac{\sqrt{m}\left(\hat{R}(t)_{R}-R_{o}\right)}{\sqrt{\operatorname{Var}\left(\hat{R}(t)_{R}\right)}}\right)^{2}$

Under $H_{o}, L_{m-R(t)}$ converges to central $\chi^{2}$ distribution with 1 degree of freedom as $m \rightarrow$ $\infty$ while under the local alternative of the form

$H_{m}: R(t)=R_{o}+\frac{\delta_{R(t)}}{\sqrt{m}}$

$L_{m-R(t)}$ converges to non-central $\chi^{2}$ distribution with non-centrality parameter

$$
\Delta_{R(t)}^{2}=\left(\frac{\sqrt{m}\left(R(t)-R_{o}\right)}{\sqrt{\operatorname{Var}\left(\hat{R}(t)_{R}\right)}}\right)^{2}
$$


Based on the asymptotic distribution of $L_{m-R(t)}$, the critical region is given by $L_{m-R(t)}>$ $\chi_{1}^{2}(\varepsilon)$ where $\varepsilon$ is the level of significance. Thus, we define PTE of $R(t)$ as

$\hat{R}(t)_{R}^{P T}=\hat{R}(t)_{R}-\left(\hat{R}(t)_{R}-R_{o}\right) I\left(L_{m-R(t)}<\chi_{1}^{2}(\varepsilon)\right)$

Finally, by invariance property of MLE, the MLE of the reliability function $P$ under this approach is given by

$\widehat{P}_{R}=\frac{{\widehat{k_{2}}}_{R}}{{\widehat{k_{1}}}_{R}+{\widehat{k_{2}}}_{R}}$

where ${\widehat{k_{1}}}_{R}=\frac{m(n+1)}{\sum_{j=1}^{m} \log \left(1+\frac{R_{j n}^{\hat{c}}}{\hat{\alpha}}\right)}$ and ${\widehat{k_{2}}}_{R}=\frac{m(n+1)}{\sum_{j=1}^{m} \log \left(1+\frac{R_{j r}^{*} \hat{c}}{\hat{\alpha}}\right)}$ such that we have $m$ independent samples of $r+1$ upper record values $R_{j 0}^{*}, \ldots, R_{j r}^{*} ; j=1,2, \ldots, m$ from three parameter Burr XII distribution. Further suppose the suspected value of $P$ is $P_{o}$ and thus we consider the following simple hypothesis to check the validity of this information:

$$
\begin{aligned}
& H_{o}: P=P_{o} \\
& H_{1}: P \neq P_{o}
\end{aligned}
$$

Under $H_{O}, \frac{\sqrt{m}\left(\hat{P}_{R}-P_{o}\right)}{\sqrt{\operatorname{Var}\left(\hat{P}_{R}\right)}} \stackrel{\text { asymp }}{\longrightarrow} N(0,1)$ and the test statistic is defined as

$L_{m-P}=\left(\frac{\sqrt{m}\left(\hat{P}_{R}-R_{o}\right)}{\sqrt{\operatorname{Var}\left(\hat{P}_{R}\right)}}\right)^{2}$

Under $H_{o}, L_{m-P}$ converges to central $\chi^{2}$ distribution with 1 degree of freedom as $m \rightarrow$ $\infty$ while under the local alternative of the form

$H_{m}: P=P_{o}+\frac{\delta_{P}}{\sqrt{m}}$

$L_{m-P}$ converges to non-central $\chi^{2}$ distribution with non-centrality parameter

$$
\Delta_{P}^{2}=\left(\frac{\sqrt{m}\left(P-P_{o}\right)}{\sqrt{\operatorname{Var}\left(\hat{P}_{R}\right)}}\right)^{2}
$$

Based on the asymptotic distribution of $L_{m-P}$, the critical region is given by $L_{m-P}>\chi_{1}^{2}(\varepsilon)$. Thus, we define PTE of $P$ as

$\hat{P}_{R}^{P T}=\hat{P}_{R}-\left(\hat{P}_{R}-P_{o}\right) I\left(L_{m-P}<\chi_{1}^{2}(\varepsilon)\right)$

\subsection{Asymptotic Bias and MSE}


We now derive the asymptotical distributional bias (ADB) and asymptotical distributional mean square error (ADMSE) of the proposed estimators in (3.1) and (3.4) under the local alternative given by (3.3). Following Saleh (2006), for any estimator $\hat{\theta}$ of $\theta$ we consider the following definitions of ADB (B) and ADMSE (M):

$$
\begin{aligned}
& B(\hat{\theta})=\lim _{m \rightarrow \infty} E[\sqrt{m}(\hat{\theta}-\theta)] \\
& M(\hat{\theta})=\lim _{m \rightarrow \infty} E\left[(\sqrt{m}(\hat{\theta}-\theta))^{2}\right]
\end{aligned}
$$

Lemma 3.1 (Saleh, 2006): If $Z \sim N(\Delta, 1)$ and $\varphi($.$) is a Borel measurable function, then$

1. $\mathrm{E}\left[\mathrm{Z} \cdot \varphi\left(\mathrm{Z}^{2}\right)\right]=\Delta \mathrm{E}\left[\varphi\left(\chi_{3}^{2}\left(\Delta^{2}\right)\right)\right]$

2. $\mathrm{E}\left[\mathrm{Z}^{2} \cdot \varphi\left(\mathrm{Z}^{2}\right)\right]=\mathrm{E}\left[\varphi\left(\chi_{3}^{2}\left(\Delta^{2}\right)\right)\right]+\Delta^{2} \mathrm{E}\left[\varphi\left(\chi_{5}^{2}\left(\Delta^{2}\right)\right)\right]$

where $\chi_{d}^{2}\left(\Delta^{2}\right)$ is the non-central $\chi^{2} r v$ with $d$ degrees of freedom and non centrality parameter $\Delta^{2}$.

From (3.13), the ADB of $\hat{k}_{R}$ and $\hat{k}_{R}^{P T}$ are obtained as follows:

$$
\begin{aligned}
B_{1}\left(\hat{k}_{R}\right) & =0 \\
B_{2}\left(\hat{k}_{R}^{P T}\right) & =\lim _{m \rightarrow \infty} E\left[\sqrt{m}\left(\hat{k}_{R}^{P T}-k\right)\right] \\
& =\lim _{m \rightarrow \infty} E\left[\sqrt{m}\left(\hat{k}_{R}-\left(\hat{k}_{R}-k_{o}\right) I\left(L_{m}<\chi_{1}^{2}(\varepsilon)\right)-k\right)\right] \\
& =\lim _{m \rightarrow \infty}\left[-E\left\{\sqrt{m}\left(\hat{k}_{R}-k_{o}\right) I\left(L_{m}<\chi_{1}^{2}(\varepsilon)\right)\right\}\right] \\
& \left.=-\sqrt{\operatorname{Var}\left(\hat{k}_{R}\right)} \lim _{m \rightarrow \infty} E\left[\frac{\sqrt{m}\left(\hat{k}_{R}-k_{o}\right)}{\sqrt{\operatorname{Var}\left(\hat{k}_{R}\right)}} I\left(\frac{\sqrt{m}\left(\hat{k}_{R}-k_{o}\right)}{\sqrt{\operatorname{Var}\left(\hat{k}_{R}\right)}}\right)^{2}<\chi_{1}^{2}(\varepsilon)\right)\right]
\end{aligned}
$$

Since $\left(\frac{\sqrt{m}\left(\hat{k}_{R}-k_{o}\right)}{\sqrt{\operatorname{Var}\left(k_{R}\right)}}\right) \stackrel{\text { asymp }}{\longrightarrow} N\left(\Delta_{k}, 1\right)$, then by applying Lemma 3.1, taking $\varphi($.$) to be an indicator$ function, we have

$$
B_{2}\left(\hat{k}_{R}^{P T}\right)=-\delta_{k} H_{3}\left(\chi_{1}^{2}(\varepsilon), \Delta_{k}^{2}\right)
$$

where $H_{d}\left(., \Delta^{2}\right)$ is the $c d f$ of non-central $\chi^{2}$ distribution with $d$ degrees of freedom and noncentrality parameter $\Delta^{2}$.

Also, for ADMSE of $\hat{k}_{R}$ and $\hat{k}_{R}^{P T}$ we get

$$
M_{1}\left(\hat{k}_{R}\right)=\lim _{m \rightarrow \infty} E\left[\left(\sqrt{m}\left(\hat{k}_{R}-k\right)\right)^{2}\right]=\operatorname{Var}\left(\hat{k}_{R}\right)
$$

and

$M_{2}\left(\hat{k}_{R}^{P T}\right)=\lim _{m \rightarrow \infty} E\left[\left(\sqrt{m}\left(\hat{k}_{R}^{P T}-k\right)\right)^{2}\right]$ 


$$
\begin{aligned}
& =\operatorname{Var}\left(\hat{k}_{R}\right) \\
& \left.+\operatorname{Var}\left(\hat{k}_{R}\right) \lim _{m \rightarrow \infty} E\left[\left(\frac{\sqrt{m}\left(\hat{k}_{R}-k_{o}\right)}{\sqrt{\operatorname{Var}\left(\hat{k}_{R}\right)}}\right)^{2} I\left(\frac{\sqrt{m}\left(\hat{k}_{R}-k_{o}\right)}{\sqrt{\operatorname{Var}\left(\hat{k}_{R}\right)}}\right)^{2}<\chi_{1}^{2}(\varepsilon)\right)\right] \\
& -2 \lim _{m \rightarrow \infty} E\left[\sqrt{m}\left(\hat{k}_{R}-k\right)\left\{\sqrt{m}\left(\left(\hat{k}_{R}-k_{o}\right) I\left(L_{m-k}<\chi_{1}^{2}(\varepsilon)\right)\right)\right\}\right] \\
& =\operatorname{Var}\left(\hat{k}_{R}\right) \\
& \left.+\operatorname{Var}\left(\hat{k}_{R}\right) \lim _{m \rightarrow \infty} E\left[\left(\frac{\sqrt{m}\left(\hat{k}_{R}-k_{o}\right)}{\sqrt{\operatorname{Var}\left(\hat{k}_{R}\right)}}\right)^{2} I\left(\frac{\sqrt{m}\left(\hat{k}_{R}-k_{o}\right)}{\sqrt{\operatorname{Var}\left(\hat{k}_{R}\right)}}\right)^{2}<\chi_{1}^{2}(\varepsilon)\right)\right] \\
& +2 \operatorname{Var}\left(\hat{k}_{R}\right) \lim _{m \rightarrow \infty}\left(\frac{\sqrt{m}\left(k-k_{o}\right)}{\sqrt{\operatorname{Var}\left(\hat{k}_{R}\right)}}\right) E\left[\left(\frac{\sqrt{m}\left(\hat{k}_{R}-k\right)}{\sqrt{\operatorname{Var}\left(\hat{k}_{R}\right)}}\right) I\left(L_{m-k}<\chi_{1}^{2}(\varepsilon)\right)\right] \\
& -2 \operatorname{Var}\left(\hat{k}_{R}\right) \lim _{m \rightarrow \infty} E\left[\left(\frac{\sqrt{m}\left(\hat{k}_{R}-k\right)}{\sqrt{\operatorname{Var}\left(\hat{k}_{R}\right)}}\right)^{2} I\left(L_{m-k}<\chi_{1}^{2}(\varepsilon)\right)\right]
\end{aligned}
$$

From Lemma 3.1 we get:

$M_{2}\left(\hat{k}_{R}^{P T}\right)=\operatorname{Var}\left(\hat{k}_{R}\right)\left[1-H_{3}\left(\chi_{1}^{2}(\varepsilon), \Delta_{k}{ }^{2}\right)+{\Delta_{k}}^{2}\left\{2 H_{3}\left(\chi_{1}^{2}(\varepsilon), \Delta_{k}{ }^{2}\right)-H_{5}\left(\chi_{1}^{2}(\varepsilon), \Delta_{k}{ }^{2}\right)\right\}\right]$

On similar lines we obtain from (3.13), the ADB of $\hat{R}(t)_{R}$ and $\hat{R}(t)_{R}^{P T}$ as follows:

$$
\begin{aligned}
B_{1}\left(\hat{R}(t)_{R}\right) & =0 \\
B_{2}\left(\hat{R}(t)_{R}^{P T}\right) & =\lim _{m \rightarrow \infty} E\left[\sqrt{m}\left(\hat{R}(t)_{P T-R}-R(t)\right]\right. \\
& =-\delta_{R(t)} H_{3}\left(\chi_{1}^{2}(\varepsilon), \Delta_{R(t)^{2}}\right)
\end{aligned}
$$

Also, for ADMSE of $\hat{R}(t)_{R}$ and $\hat{R}(t)_{R}^{P T}$ we get

$M_{1}\left(\hat{R}(t)_{R}\right)=\lim _{m \rightarrow \infty} E\left[\left(\sqrt{m}\left(\hat{R}(t)_{R}-R(t)\right)\right)^{2}\right]=\operatorname{Var}\left(\hat{R}(t)_{R}\right)$

and from Lemma 3.1

$$
\begin{aligned}
M_{2}\left(\hat{R}(t)_{R}^{P T}\right) & =\lim _{m \rightarrow \infty} E\left[\left(\sqrt{m}\left(\hat{R}(t)_{P T-R}-R(t)\right)\right)^{2}\right] \\
& =\operatorname{Var}\left(\hat{R}(t)_{R}\right)\left[1-H_{3}\left(\chi_{1}^{2}(\varepsilon), \Delta_{R(t)}{ }^{2}\right)+\Delta_{R(t)}{ }^{2}\left\{2 H_{3}\left(\chi_{1}^{2}(\varepsilon), \Delta_{R(t)}{ }^{2}\right)-H_{5}\left(\chi_{1}^{2}(\varepsilon), \Delta_{R(t)}{ }^{2}\right)\right\}\right]
\end{aligned}
$$

Finally, the ADB of $\hat{P}_{R}$ and $\hat{P}_{R}^{P T}$ are obtained as follows:

$$
\begin{aligned}
B_{1}\left(\hat{P}_{R}\right) & =0 \\
B_{2}\left(\hat{P}_{R}^{P T}\right) & =\lim _{m \rightarrow \infty} E\left[\sqrt{m}\left(\hat{P}_{P T-R}-P\right)\right] \\
& =-\delta_{P} H_{3}\left(\chi_{1}^{2}(\varepsilon), \Delta_{P}{ }^{2}\right)
\end{aligned}
$$

Also, for ADMSE of $\hat{P}_{R}$ and $\hat{P}_{R}^{P T}$ we get

$M_{1}\left(\hat{P}_{R}\right)=\lim _{m \rightarrow \infty} E\left[\left(\sqrt{m}\left(\hat{P}_{R}-P\right)\right)^{2}\right]=\operatorname{Var}\left(\hat{P}_{R}\right)$

and from Lemma 3.1

$$
\begin{aligned}
M_{2}\left(\hat{P}_{R}^{P T}\right) & =\lim _{m \rightarrow \infty} E\left[\left(\sqrt{m}\left(\hat{P}_{P T-R}-P\right)\right)^{2}\right] \\
& =\operatorname{Var}\left(\hat{P}_{R}\right)\left[1-H_{3}\left(\chi_{1}^{2}(\varepsilon), \Delta_{P}^{2}\right)+\Delta_{P}^{2}\left\{2 H_{3}\left(\chi_{1}^{2}(\varepsilon), \Delta_{P}^{2}\right)-H_{5}\left(\chi_{1}^{2}(\varepsilon), \Delta_{P}^{2}\right)\right\}\right]
\end{aligned}
$$

\subsection{Comparison}


In this section, we analyze the ADMSE of the proposed estimators to study their relative performance. From (3.4), we see that if $L_{m} \rightarrow 0$ then $\hat{k}_{R}^{P T} \rightarrow k_{o}$ while $\hat{k}_{R}^{P T} \rightarrow \hat{k}_{R}$ as $L_{m} \rightarrow$ $\infty$. The asymptotic relative efficiency (ARE) of $\hat{k}_{R}^{P T}$ over $\hat{k}_{R}$ is defined as:

$$
\begin{aligned}
\operatorname{ARE}\left(\hat{k}_{R}^{P T}, \hat{k}_{R}\right) & =\frac{M_{1}\left(\hat{k}_{R}\right)}{M_{2}\left(\hat{k}_{R}^{P T}\right)} \\
& =\left[1-H_{3}\left(\chi_{1}^{2}(\varepsilon), \Delta_{k}{ }^{2}\right)+\Delta_{k}{ }^{2}\left\{2 H_{3}\left(\chi_{1}^{2}(\varepsilon), \Delta_{k}{ }^{2}\right)-H_{5}\left(\chi_{1}^{2}(\varepsilon), \Delta_{k}{ }^{2}\right)\right\}\right]^{-1}
\end{aligned}
$$

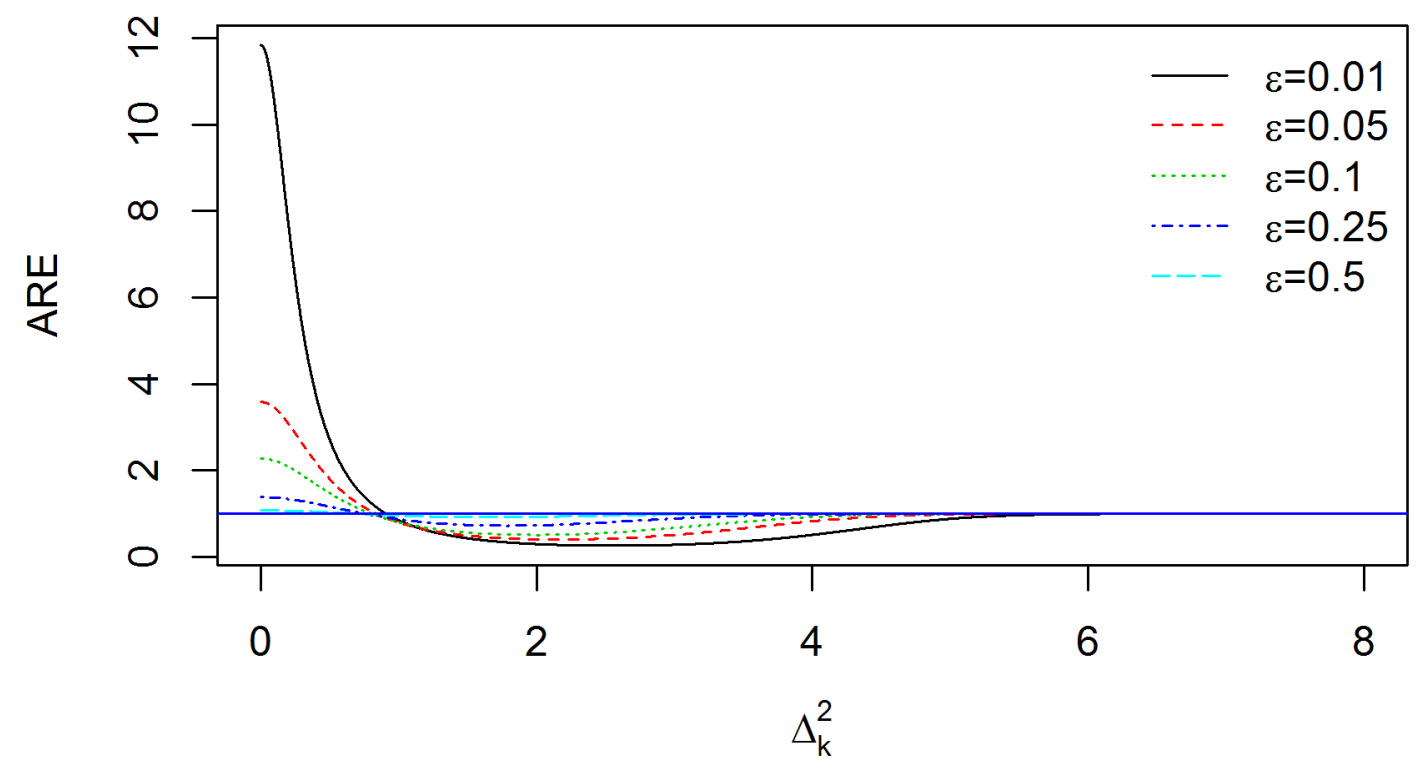

Figure 14: Asymptotic Relative Efficiency of $\hat{k}_{R}^{P T}$ over $\hat{k}_{R}$

Figure 14 shows the asymptotic relative efficiency of $\hat{k}_{R}^{P T}$ for different values of $\varepsilon$ verses $\Delta_{k}{ }^{2}$. We observe that the relative efficiencies have a peak at point zero, then it decreases and gets a minimum at some values of $\Delta_{k}{ }^{2}$. Then the relative efficiencies increase to line 1. Also, it is seen that the proposed PTE have the highest relative efficiency for the smallest values of $\varepsilon$. Further, as it increases the maximum relative efficiencies decrease while the minimum relative efficiencies increase. Finally, we can conclude that $\hat{k}_{R}^{P T}$ is a better estimator of $k$ than $\hat{k}_{R}$ whenever:

$$
0 \leq \Delta_{k}^{2} \leq \frac{H_{3}\left(\chi_{1}^{2}(\varepsilon), \Delta_{k}^{2}\right)}{2 H_{3}\left(\chi_{1}^{2}(\varepsilon), \Delta_{k}^{2}\right)-H_{5}\left(\chi_{1}^{2}(\varepsilon), \Delta_{k}^{2}\right)}
$$

By the asymptotic normality of MLE for the parameters $c$ and $\alpha$, one may obtain similar results. On similar lines, the ARE of $\hat{R}(t)_{R}^{P T}$ over $\hat{R}(t)_{R}$ is defined as $\operatorname{ARE}\left(\hat{R}(t)_{R}^{P T}, \hat{R}(t)_{R}\right)=\left[1-H_{3}\left(\chi_{1}^{2}(\varepsilon), \Delta_{R(t)}{ }^{2}\right)+\Delta_{R(t)}{ }^{2}\left\{2 H_{3}\left(\chi_{1}^{2}(\varepsilon), \Delta_{R(t)}{ }^{2}\right)-H_{5}\left(\chi_{1}^{2}(\varepsilon), \Delta_{R(t)}{ }^{2}\right)\right\}\right]^{-1}$ 
and we can conclude that $\hat{R}(t)_{R}^{P T}$ is a better estimator of $R(t)$ than $\hat{R}(t)_{R}$ whenever

$$
0 \leq \Delta_{R(t)}{ }^{2} \leq \frac{H_{3}\left(\chi_{1}^{2}(\varepsilon), \Delta_{R(t)}{ }^{2}\right)}{2 H_{3}\left(\chi_{1}^{2}(\varepsilon), \Delta_{R(t)}{ }^{2}\right)-H_{5}\left(\chi_{1}^{2}(\varepsilon), \Delta_{R(t)}{ }^{2}\right)}
$$

Similarly, $\hat{P}_{R}^{P T}$ is a better estimator of $P$ than $\hat{P}_{R}$ whenever

$$
0 \leq \Delta_{P}^{2} \leq \frac{H_{3}\left(\chi_{1}^{2}(\varepsilon), \Delta_{P}^{2}\right)}{2 H_{3}\left(\chi_{1}^{2}(\varepsilon), \Delta_{P}^{2}\right)-H_{5}\left(\chi_{1}^{2}(\varepsilon), \Delta_{P}^{2}\right)}
$$

\section{An Example on Real Data}

To illustrate the estimation methods proposed in the preceding sections, we consider data on maximum flood level (in millions of cubic feet per second) for the Susquehanna River of Harrisburg over 20 four-year periods. This data was considered by Dumonceaux and Antle 1973 and is as follows:

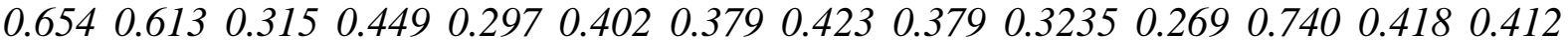
0.4940 .4160 .3380 .3920 .4840 .265

Kolmogorov Smirnov test generated the test statistic $D=0.14$ and a $p$-value of 0.8996 which indicates that the data fits the three parameter Burr distribution. Using an iterative algorithm, we obtained the MLES of the parameters as $\hat{k}=0.5829, \hat{c}=8.3113$, $\hat{\alpha}=0.0002$. Thus, the reliability function $R(t)=0.8899$ at $t=0.3$. The following are the upper record values obtained from the sample.

0.65400 .7400

Based on these record values, we obtain the preliminary test estimators of $k$ and $R(t)$ for various test values of $k_{o}$ and $R_{o}(t)$ respectively. The results are shown in Tables 1 and 2 respectively.

Table 1: Preliminary test estimators of $k$

\begin{tabular}{cccc}
\hline$k_{o}$ & $\hat{k}_{M L}^{P T}$ & $\hat{k}_{U}^{P T}$ & $\hat{k}_{M R}^{P T}$ \\
\hline 0.10 & 0.1000 & 0.1000 & 0.1000 \\
0.20 & 0.2000 & 0.2000 & 0.2000 \\
0.30 & 0.3000 & 0.3000 & 0.3000 \\
0.40 & 0.4000 & 0.4000 & 0.4000 \\
0.50 & 0.5000 & 0.5000 & 0.5000 \\
0.60 & 0.6000 & 0.6000 & 0.6000 \\
0.70 & 0.7000 & 0.7000 & 0.7000 \\
0.80 & 0.8000 & 0.8000 & 0.8000 \\
0.90 & 0.9000 & 0.9000 & 0.9000 \\
1.00 & 0.3334 & 0.1667 & 0.0000 \\
\hline
\end{tabular}


Table 2: Preliminary test estimators of $R(t)$

\begin{tabular}{ccc}
\hline$R_{o}(t)$ & $\hat{R}(t)^{P T}$ & $\tilde{R}(t)^{P T}$ \\
\hline 0.10 & 0.9355 & 0.9667 \\
0.30 & 0.9355 & 0.9667 \\
0.50 & 0.9355 & 0.9667 \\
0.70 & 0.9355 & 0.9667 \\
0.80 & 0.9355 & 0.9667 \\
0.90 & 0.9000 & 0.9000 \\
0.93 & 0.9300 & 0.9300 \\
0.95 & 0.9500 & 0.9500 \\
0.97 & 0.9700 & 0.9700 \\
1.00 & 0.9355 & 0.9667 \\
\hline
\end{tabular}

\section{Conclusion}

We have proposed various preliminary test estimators for estimation of the powers of the parameter $k$ and reliability functions of three parameters Burr XII distribution under the assumption of known values of parameters $c$ and $\alpha$. The exact bias and MSE expressions have been derived. We have also discussed a case when all the parameters of the distribution are unknown. It can be concluded that all of the proposed PTES dominate their corresponding usual estimators such as UMVUE, MLE, MR and EBE in the neighbourhood of null hypothesis $H_{o}: k=k_{o}$. The relative efficiency of PTES of the powers of the parameter $k$ is higher when $k$ is close to its hypothesized value $k_{o}$. Similarly, PTES of $R(t)$ and $P(X>$ $Y$ ) perform better than their usual estimators whenever the true value of the parameter is close to its hypothetical value.

Next, we have also developed improved preliminary test confidence intervals of the parameter $k$ and have shown them to have a greater coverage probability and a smaller expected length compared to the usual equal tail confidence intervals whenever $k$ is sufficiently close to $k_{o}$. Thus, we were able to establish improved estimators and confidence intervals of the parametric functions of the three parameters Burr XII distribution.

\section{Acknowledgement}

The authors would like to thank all the Reviewers for their valuable comments and suggestions that improved the presentation of the paper.

\section{References}


1. Arnold, B. C., Balakrishan, N., Nagaraja, H. N. (1992): A First Course in Order Statistics. John Wiley \& Sons, New York.

2. Arashi,M., Emadi, M. (2008): Evidential inference based on record data and inter-record times. Stat.Papers, 13(8), 380-210.

3. Balakrishan, N., Ahsanullah, M.,Chan, P.S. (1995): On the logistic record values and associated inference. Journal of Applied Statistical Science, 2, 233-248.

4. Bancroft, T. A. (1944): On biases in estimation due to use of preliminary tests of significance. Ann. Math., 15, 190-204.

5. Belaghi, R. A., Arashi, M., Tabatabaey, S. M. M. (2014): Improved confidence intervals for the scale parameter of Burr XII model based on record values. Comput. Stat., DOI 10.1007/s00180-014-0484-3.

6. Belaghi, R. A., Arashi, M., Tabatabaey, S. M. M. (2015): On the Construction of Preliminary Test Estimator Based on Record Values for the Burr XII Model. Communications in Statistics - Theory and Methods, 44(1), 1-23, DOI:10.1080/03610926.2012.733473

7. Belaghi, R. A., Noori, A. M. (2016): Estimation based on progressively type-I hybrid censored data from the Burr XII distribution. Statistical Papers, DOI: 10.1007/s00362-0160849-5.

8. Belaghi, R. A., Noori, A. M., Bevrani, H. (2017): Estimation on Burr XII under Progressive hybrid type I censored data, Methodology and Computing in Applied Probability, 9(2), 665683.

9. Burr, I. W (1942): Cumulative Frequency Functions. The Annals of Mathematical Statistics 13 (2), 215-232

10. Chandler, K. N. (1952): The distribution and frequency of record values. Journal of the Royal Statistical Society, Series B, 14, 220-228.

11. Chaturvedi, A., Malhotra, A. (2016): Estimation and Testing Procedures for the Reliability Functions of a Family of Lifetime Distributions based on Records. Int J Syst Assur Eng Manag, DOI:10.1007/s13198-016-0531-2

12. Chaturvedi, A., Malhotra, A. (2017): Inference on the Parameters and Reliability Characteristics of three parameter Burr Distribution based on Records. Appl. Math. Inf. Scien., 11(3), 837-849.

13. Constantine, K., Karson, M. and Tse, S. K. (1986): Estimation of $\mathrm{P}(\mathrm{Y}<\mathrm{X})$ in the gamma case. Commun. Statist. - Simul., 15(2), 365-388. 
14. Dumonceaux, R., Antle, C.E. (1973): Discrimination between the lognormal and Weibull distributions. Technometrics, 15, 923-926.

15. Glick, N. (1978): Breaking records and breaking boards. American Mathematical Monthly, 85, 543-551.

16. Gulati, S., Padgett, W. J. (1991): Smooth nonparametric estimation of the distribution and density function from record breaking data. Statistical Technical Report, University Of South Carolina.

17. Gulati, S., Padgett, W. J. (1994): Smooth nonparametric estimation of the hazard and hazard rate function from record breaking data. J. Stat. Plann. Inf. 42, 331-341.

18. Gulati, S., Padgett, W. J. (1995): Nonparamertic function estimation from inversely sampled record breaking data. Canad. J. Stat. 23(4), 359-368.

19. Habibi Rad, A., Arghami, N. R., Ahmadi, J. (2006): Statistical evidence in experiments and in record values. Commun. Stat. Theo. Meth. 35(11), 1971-1983.

20. Hogg, R. V., Klugman, S. A. (1984): Loss Distributions. John Wiley \& Sons, Inc.

21. Nagaraja, H. N. (1988a): Record values and related statistics - A review. Communictions in Statistics - Theory and Methods, 17, 2223-2238.

22. Nagaraja, H. N. (1988b): Some characterizations of continuous distributions based on regressions of adjacent order statistics and record values. Sankhya, Series A, 50, 70-73.

23. Razmkhah, M., Ahmadi, J. (2011): Comparing two sampling schemes based on entropy of record statistics. Stat. Papers, 53, 95-106

24. Saleh, A. K. M. E. (2006): Theory of Preliminary Test and Stein-type Estimations with Applications. New York: Wiley.

25. Saleh, A. K. M. E., Kibria, B. M. G. (1993): Performance of some new preliminary test ridge regression estimators and their properties. Comm. Stat. - Theo. Meth., 22(10), 2747-2764.

26. Saleh, A. K. M. E., Sen, P. K. (1978): Nonparametric estimation of location parameter after a preliminary test on regression. Ann. Stat., 6, 154-168.

27. Shao, Q. (2004): Notes on maximum likelihood estimation for the three-parameter Burr XII distribution. Comp. Stat. Data Anal., 45, 675-687.

28. Watson, R. I. (1952): Research design and methodology in evaluating the results of psychotherapy. J. Clin. Psychol., 8, 29-33. DOI:10.1002/1097-4679(195201)8:1<29::AIDJCLP2270080107>3.0.CO;2-O 
